\title{
Petrogénesis y condiciones de cristalización del domo intracratérico del volcán Cerro Bravo, Colombia
}

\author{
Camilo Pinzón $^{1 *}$; Juan Felipe Echeverrii ${ }^{1}$; Hugo Murcia $^{2,3}$; Dayana Schonwalder-Ángel ${ }^{4}$
}

DOI: http://dx.doi.org/10.18273/revbol.v40n3-2018004@ @

Forma de citar: Pinzón, C., Echeverri, J.F., Murcia, H., y Schonwalder-Ángel, D. (2018). Petrogénesis y condiciones de cristalización del domo intracratérico del volcán Cerro Bravo, Colombia. Boletín de Geología, 40(3), 67-84. DOI: 10.18273/revbol.v40n3-2018004.

Material suplementario electrónico: La versión en línea de este artículo contiene MATERIAL SUPLEMENTARIO.

\section{RESUMEN}

El volcán Cerro Bravo (VCB) es un volcán compuesto ubicado en la Cordillera Central de Colombia. Durante los 50 ka de evolución del volcán la actividad eruptiva ha variado entre erupciones plinianas y emisiones efusivas. Actualmente, el cráter se encuentra ocupado por un domo que representa la última fase eruptiva de la erupción más reciente. El domo presenta una mineralogía típica de rocas andesíticas-dacíticas, con cristales de plagioclasa, anfíbol y piroxeno. La composición del anfíbol evidencia condiciones de cristalización entre 1 y 3,5 kba, de 800 a $950^{\circ} \mathrm{C}$, de $-5,3$ a $-6,8 \mathrm{fO}_{2}$, y de 5,1 a 6,8 $\mathrm{H}_{2} \mathrm{O}$ wt.\% en el fundido, mientras que en el piroxeno evidencia condiciones de 914 $\pm 97^{\circ} \mathrm{C}$ y $18,5 \pm 9,2 \mathrm{Kba}$. Con base en estos resultados y en las relaciones mineralógicas, se puede establecer una historia de cristalización de la siguiente manera: Augita y enstatita fueron los primeros minerales en cristalizar ( $>20$ - 30 km). Posteriormente hubo un ascenso hacia una cámara magmática ubicada entre 4,6 y 13,2 km donde comenzó la cristalización del anfíbol (a la base de la cámara cristalizó pargasita, y al techo edenita y magnesihornblenda). La disminución en la temperatura, presión y el contenido de calcio durante el ascenso del magma favorecieron la cristalización de labradorita, la cual fue variando hasta andesina. Los microlitos que hacen parte de la masa fundamental son evidencia de las últimas fases de cristalización producto de la descompresión magmática. Este estudio muestra las condiciones de cristalización que representan la última fase eruptiva de la erupción más reciente del VCB.

Palabras clave: Anfíbol; geotermobarometría; cámaras magmática; evolución magmática; volcán poligenético.

\section{Petrogenesis and crystallisation conditions of the intra-crater dome of Cerro Bravo volcano, Colombia}

\begin{abstract}
Cerro Bravo is a composite volcano located in the Central Cordillera of Colombia. Throughout its 50 ka of evolution, this volcano has been characterised by having successive explosive to effusive phases. At present, the crater hosts a dome that represents the last eruptive phase of the last eruption. The dome has a typical mineralogy of andesite-dacite rocks with plagioclase, amphibole and pyroxene crystals. The amphibole composition allow us to constrain the melt crystallisation conditions at 1 to $3.5 \mathrm{kba}, 800$ to $950^{\circ} \mathrm{C}$, -5.3 to $-6.8 \mathrm{fO}_{2}$, and 5.1 to $6.8 \mathrm{H}_{2} \mathrm{O}$ wt. \%, while the piroxene composition at $914 \pm 97^{\circ} \mathrm{C}$ y $18,5 \pm 9,2 \mathrm{Kba}$. Based on these results and the mineralogical configuration, it is possible to establish a crystallisation history as follows: Augite and enstatite were the first formed minerals $(>20-30 \mathrm{~km})$. After that, the magma ascended up to a magma chamber located between 4.6 and $13.2 \mathrm{~km}$ from the surface where the amphibole crystallisation started (pargasite at the base of the chamber and edenite and magnesiohornblende at the top). The drop in temperature and pressure also promoted the crystallisation of labradorite that transitionally changed to andesine. Plagioclase microlites in the groundmass are evidence of the last crystallisation phase close the surface, as a result of magmatic decompression. This study shows the crystalisation conditions that represent the last eruptive phase of the last eruption of the Cerro Bravo volcano.
\end{abstract}

Keywords: Amphibole; geothermobarometry; magma chamber; magmatic evolution; polygenetic volcano.

\footnotetext{
${ }^{1}$ Programa de Geología, Universidad de Caldas, Manizales, Colombia. (*) camilo.geology@gmail.com; pipeche02@gmail.com ${ }^{2}$ Departamento de Ciencias Geológicas, Universidad de Caldas, Manizales, Colombia. hugo.murcia@ucaldas.edu.co

${ }^{3}$ Instituto de Investigaciones en Estratigrafía, Universidad de Caldas, Manizales, Colombia.

${ }^{4}$ Earth Observatory of Singapore, Nanyang Technological University, Singapore, Singapore. angel.d@ntu.edu.sg
} 


\section{INTRODUCCIÓN}

El volcán Cerro Bravo (VCB; N 0505’26,06”, W 75¹7’33,14”, 4000 msnm) es un volcán compuesto de composición dacítica y andesítica (Lescinsky, 1990), ubicado en la parte central de la Provincia Volcano Tectónica San Diego - Cerro Machín (PVTSC; Martínez et al., 2014) (FIGURA 1). La edad más antigua reportada para el VCB es $50 \pm 50$ ka (método K/Ar; Lescinsky, 1990), y su historia eruptiva se caracteriza por haber presentado eventos de crecimiento y destrucción de domos (Lescinsky, 1990; Monsalve, 1991; Monsalve y Nuñez, 1992).

La actividad más antigua identificada en el VCB está relacionada con la formación de la caldera de Quebrada Seca o volcán Cerro Bravo Antiguo, mientras que la actividad más reciente (14.000 años) se enmarca dentro del volcán Cerro Bravo Moderno (Lescinsky, 1990). Actualmente, el cráter del VCB está ocupado por un domo parcialmente destruido, el cual representa la actividad efusiva más reciente. Según Lescinsky (1990), la actividad del VCB ha migrado de sur a norte, y actualmente el volcán está constituido por dos edificios volcánicos. Los estilos eruptivos del VCB varían desde erupciones plinianas hasta emisiones efusivas (Calvache et al., 1987; Lescinsky, 1990; Monsalve, 1991; Monsalve y Nuñez, 1992).

En términos petrogenéticos, los volcanes de la PVTSC han sido poco estudiados $y$, por ende, son poco conocidos tanto los procesos involucrados en la evolución de los magmas como las profundidades de almacenamiento de los mismos (ver e.g. Murcia et al., en prensa). En la PVTSC solo dos estudios petrogenéticos y de condiciones de cristalización han sido realizados, los cuales corresponden al domo intracratérico del volcán Cerro Machín (Laeger et al., 2013), y a algunos productos del Complejo Volcánico Nevado del Ruiz (Rayo-Rocha y Zuluaga, 2011; Rayo-Rocha, 2012). Para una cadena volcánica activa con más de 10 centros eruptivos poligenéticos (e.g. Londoño, 2016; Murcia et al., en prensa), es obvia la escasez de este tipo de estudios que pueden indicar, por ejemplo, las condiciones y localización de reservorios o cámaras magmáticas (Stechern et al., 2017), como también procesos de mezcla de magmas que probablemente disparan erupciones (Laeger et al., 2013). Así, esta información es fundamental para la evaluación de la amenaza volcánica en general.
En este trabajo se realiza una caracterización composicional de las fases minerales del domo intracratérico del VCB con el objetivo de proveer información acerca de la petrogénesis y las condiciones de cristalización magmática a través de análisis petrográficos y de química mineral. También, por medio de métodos geotermobarométricos, se determinan condiciones de presión, temperatura, fugacidad de oxígeno y cantidades de agua en el magma que dio origen al domo. Finalmente, se establece un modelo que ilustra la dinámica en profundidad en términos de evolución magmática asociada con la última fase de la última erupción del VCB.

\section{MARCO GEOLÓGICO-ESTRUCTURAL}

El Cinturón Volcánico de Los Andes se encuentra asociado a la subducción de las placas Nazca y Caribe bajo el borde NW de la placa Suramericana (Bourdon et al., 2003). Así, el magmatismo asociado a este margen continental, ha estado activo desde el Plio - Cuaternario (Bourdon et al., 2003). Este cinturón está dividido en cuatro segmentos volcánicos (Jordan et al., 1983) (FIGURA 1A), siendo el segmento más septentrional correspondiente a la Zona Volcánica Norte (ZVN, Thorpe et al., 1984; Stern et al., 1984; Bourdon et al., 2003) (FIGURA 1A). En este segmento, al norte, se encuentra la PVTSC (FIGURA 1B) y en el centro de ésta, el volcán Cerro Bravo (FIGURA 1C).

La PVTSC está enmarcada dentro de una zona de alta deformación tectónica caracterizada por un sistema de fallas regionales constituida por el sistema de fallas Villamaría - Termales, la falla Santa Rosa y el sistema de fallas de Palestina. Este último, es el sistema que ejerce mayor control estructural en la zona y se encuentra dentro de los sistemas NNE - SSW y NE - SW de la Cadena Andina (CHEC, 1983; González y Jaramillo, 2002; Cárdenas, 2004; Cárdenas et al., 2004; Acosta et al., 2007; Mejía et al., 2012). Para estos autores, el sistema de fallas de Palestina ha capturado cuerpos magmáticos durante el Cenozoico y se ha convertido en la ruta de alimentación de los centros volcánicos en la parte más septentrional de la ZVN. El sistema de fallas Villamaría - Termales es una falla de rumbo de carácter sinextro-lateral (Thouret, 1989), con una dirección predominante N65W (González y Jaramillo, 2002), mientras que la falla Santa Rosa está definida como una falla de tipo inversa dextral (Guzmán et al., 1998). 
El VCB se encuentra sobre rocas metamórficas de edad Triásica (Villagómez et al., 2011; Villagómez y Spikings, 2013) o Jurásica Superior (Blanco-Quintero et al., 2014) pertenecientes al Complejo Cajamarca y rocas ígneas plutónicas del Batolito de El Bosque, de edad Paleoceno (Vesga y Barrero, 1978). En el área también se encuentran depósitos de la Formación Casabianca de edad Plioceno - Pleistoceno (Borrero y Naranjo, 1990).

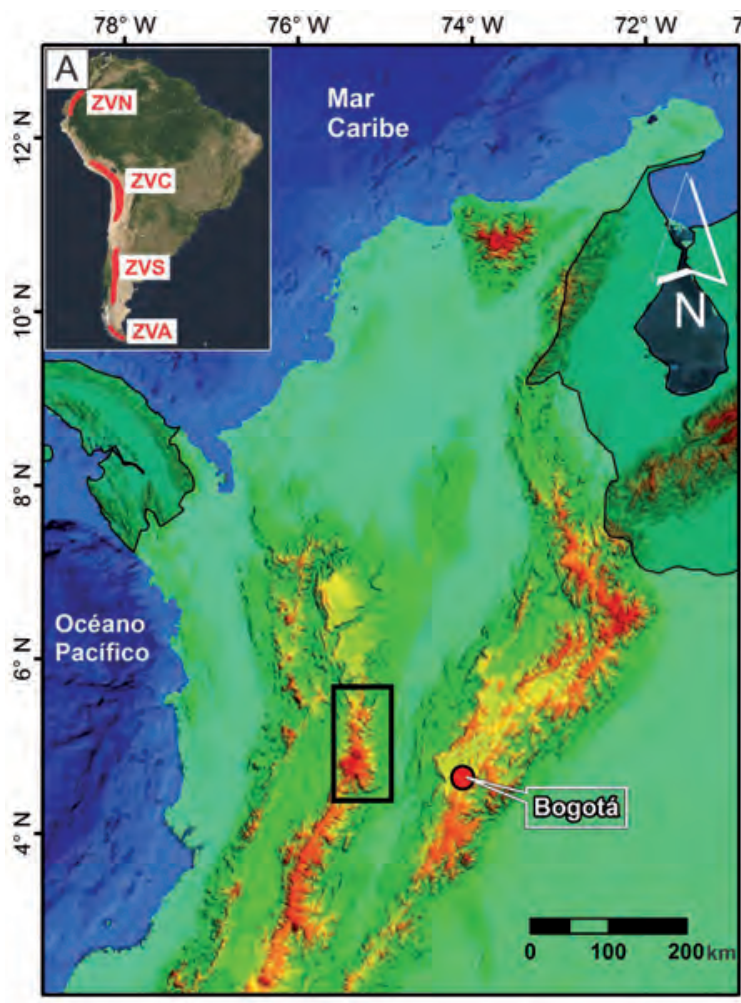

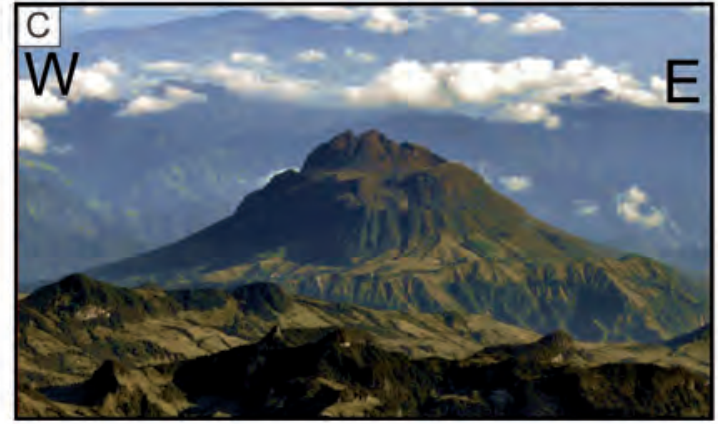

Ciudades principales $75^{\circ} 36^{\prime} \mathrm{W} \quad 75^{\circ} 30^{\prime} \mathrm{W} \quad 75^{\circ} 24^{\prime} \mathrm{W} \quad 75^{\circ} 19^{\prime} \mathrm{W} \quad 75^{\circ} 14^{\prime} \mathrm{W} \quad 75^{\circ} 8^{\prime} \mathrm{W} \quad 75^{\circ} 3^{\prime} \mathrm{W} \quad 74^{\circ} 577^{\prime} \mathrm{W}$

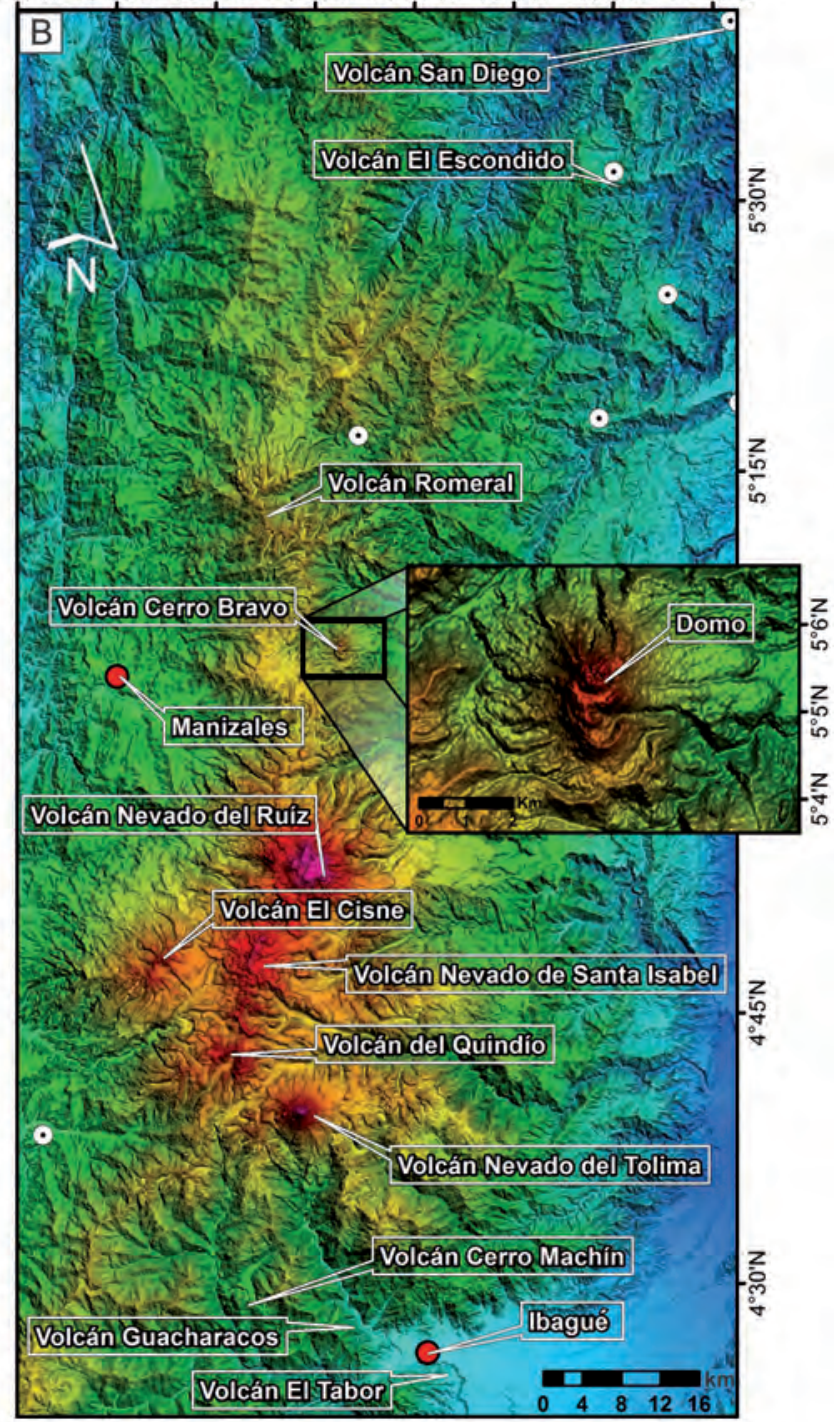

$\odot$ Cabeceras municipales

FIGURA 1. A. Localización de la Provincia Volcano Tectónica San Diego - Cerro Machín en Colombia (recuadro negro). Note en la parte superior izquierda los segmentos volcánicos de Los Andes Suramericanos; ZVN: Zona Volcánica Norte, ZVC: Zona Volcánica Centro, ZVS: Zona Volcánica Sur, ZVA: Zona Volcánica Austral. B. Volcanes pertenecientes a la Provincia Volcano Tectónica San Diego - Cerro Machín. C. Volcán Cerro Bravo (Imagen tomada de www.sgc.gov.co). 


\section{METODOLOGÍA}

Este trabajo se basa en el análisis de siete muestras de roca tomadas alrededor del domo intracratérico del VCB. En todas ellas se realizaron análisis petrográficos y de química mineral. Las secciones delgadas fueron preparadas en los laboratorios del Earth Observatory of Singapore, Nanyang Technological University (Singapore) así como de la Universidad de Caldas (Colombia). Los análisis petrográficos fueron realizados en el Instituto de Investigaciones en Estratigrafía (IIES) de la Universidad de Caldas (Colombia) e incluyeron la determinación de las características mineralógicas y texturales, además de los porcentajes de cada fase mineral y de la masa fundamental a partir de un barrido de 1 x 2 mm en cada muestra. Los análisis de química mineral se llevaron a cabo en la microsonda de emisión de campo JEOL JXA-8530F del Earth Observatory of Singapore, Nanyang Technological University (Singapore) e incluyeron 235 análisis químicos de elementos mayores de minerales de plagioclasa, anfíbol y piroxeno (MATERIAL SUPLEMENTARIO). El procesamiento y la representación gráfica de los resultados fueron llevados a cabo a través de los programas GCDkit 4,1 (Janoušek et al., 2006), CFU y CFU-PINGU (Cortés, 2015). Las abreviaciones usadas para la representación en las imágenes fueron tomadas de Whitney y Evans (2010).

\section{RESULTADOS}

\section{Descripción de la roca}

Las rocas del domo intracratérico del VCB presentan un promedio de 53 vol. $\%$ matriz, 46 vol. $\%$ cristales y 1 vol.\% vesículas. Las rocas exhiben textura porfirítica y presentan fenocristales de plagioclasa, anfíbol y piroxeno. La masa fundamental está compuesta de microlitos $(<0,05 \mathrm{~mm})$ de plagioclasa, hornblenda y piroxeno, y algunos minerales accesorios como óxidos y apatito. Estos están embebidos en una masa vítrea afanítica de color gris claro, en ocasiones con textura fluidal incipiente. La asociación mineralógica presente se compone de fenocristales $(\geq 0,5 \mathrm{~mm})$ y microfenocristales $(0,5-0,05 \mathrm{~mm})$ de plagioclasa (Pl) ( 23,5\%), anfíbol (Anf) ( 16,9\%), ortopiroxeno (Opx) ( 2,7\%) y clinopiroxeno (Cpx) ( 2,9\%). Oxihornblenda (Ohbl) constituye alrededor de 2,0\% de los anfíboles presentes (TABLA 1). En general, se observan texturas glomeroporfirítica, cumuloporfirítica y seriada.

TABLA 1. Porcentajes de tipos y tamaños de cristales, masa fundamental y vesículas presentes en las rocas analizadas del domo del VCB.

\begin{tabular}{|c|c|c|c|c|c|c|c|c|c|c|c|c|c|c|}
\hline \multirow[t]{3}{*}{ Muestra } & \multicolumn{10}{|c|}{ Mineralogía (\%) } & \multirow[t]{3}{*}{ Crist. \% } & \multirow[t]{3}{*}{ M.F. } & \multirow[t]{3}{*}{ Vesic. \% } & \multirow[t]{3}{*}{ Total } \\
\hline & \multicolumn{2}{|c|}{ Pl } & \multicolumn{2}{|c|}{ Anf } & \multicolumn{2}{|c|}{ Ohb } & \multicolumn{2}{|c|}{ Cpx } & \multicolumn{2}{|c|}{ Opx } & & & & \\
\hline & Fn & Mf & Fn & Mf & Fn & Mf & Fn & Mf & Fn & Mf & & & & \\
\hline CH1 & 9,9 & 9,9 & 6,3 & 6,3 & 0,5 & 2,3 & 1,4 & 2,7 & 0,5 & 1,4 & 41 & 58 & 1 & 100 \\
\hline $\mathrm{CH} 2$ & 5,9 & 15,1 & 7,8 & 5,0 & 0,9 & 0,5 & 1,4 & 1,4 & 3,2 & 0,9 & 42 & 57 & 1 & 100 \\
\hline $\mathrm{CH} 4$ & 9,8 & 12,9 & 5,8 & 5,8 & 0,9 & 0,9 & 0,4 & 1,3 & 0,9 & 1,3 & 40 & 59 & 1 & 100 \\
\hline CH5 & 10,9 & 12,9 & 8,4 & 6,9 & 1,5 & 1,0 & 0,5 & 0,5 & 1,5 & 2,0 & 46 & 54 & 0 & 100 \\
\hline CH6 & 14,8 & 13,7 & 9,3 & 8,8 & 0,5 & 0,0 & 2,7 & 0,5 & 1,1 & 0,5 & 52 & 48 & 0 & 100 \\
\hline $\mathrm{CH} 7$ & 16,7 & 10,9 & 11,5 & 5,8 & 1,7 & 0,6 & 0,6 & 2,3 & 0,6 & 2,3 & 53 & 46 & 1 & 100 \\
\hline CH8 & 10,8 & 10,3 & 8,8 & 7,7 & 2,6 & 0,5 & 1,0 & 2,1 & 1,5 & 2,6 & 48 & 51 & 1 & 100 \\
\hline Promedio & \multicolumn{2}{|c|}{23,5} & \multicolumn{2}{|c|}{16,9} & \multicolumn{2}{|c|}{2,0} & \multicolumn{2}{|c|}{2,7} & \multicolumn{2}{|c|}{2,9} & 46 & 53 & 1 & \\
\hline
\end{tabular}

\section{Mineralogía}

La plagioclasa es el mineral más representativo (TABLA 1). Los cristales se encuentran frescos y son principalmente subhedrales, aunque algunos son euhedrales y en menor proporción anhedrales. Se observan maclas de albita, periclina, carlsbald y albita - periclina, y zonaciones normales, inversas y oscilatorias (FIGURA 2A y B); algunos cristales presentan texturas de reabsorción y textura en tamiz. Los cristales alcanzan tamaños hasta de $2,5 \mathrm{~mm}$ y se observan también como inclusiones en anfíbol y piroxeno (FIGURA 2C), así como formando texturas glomeroporfirítica (FIGURA 2A) y cumuloporfirítica (FIGURA 2D).

El anfíbol es la siguiente fase mineralógica más abundante (TABLA 1). Los cristales son predominantemente subhedrales, y solo algunos son euhedrales. Se observan cristales con cortes longitudinales típicos con hábito prismático (FIGURA 
2C), y cortes basales que presentan dos direcciones de clivaje. Algunos fenocristales de hornblenda presentan maclas simples y contienen inclusiones de plagioclasa (FIGURA 2C), mientras que otros cristales exhiben una textura esqueletal. Los cristales alcanzan tamaños hasta de $2,2 \mathrm{~mm}$. Se observan en texturas cumuloporfirítica (FIGURA 2D) y glomeroporfirítica. Cristales de oxihornblenda, se reconocen por su fuerte pleocroísmo (marrón a marrón rojizo) y bordes oscuros, así como sus bordes de reacción (FIGURA 2E).
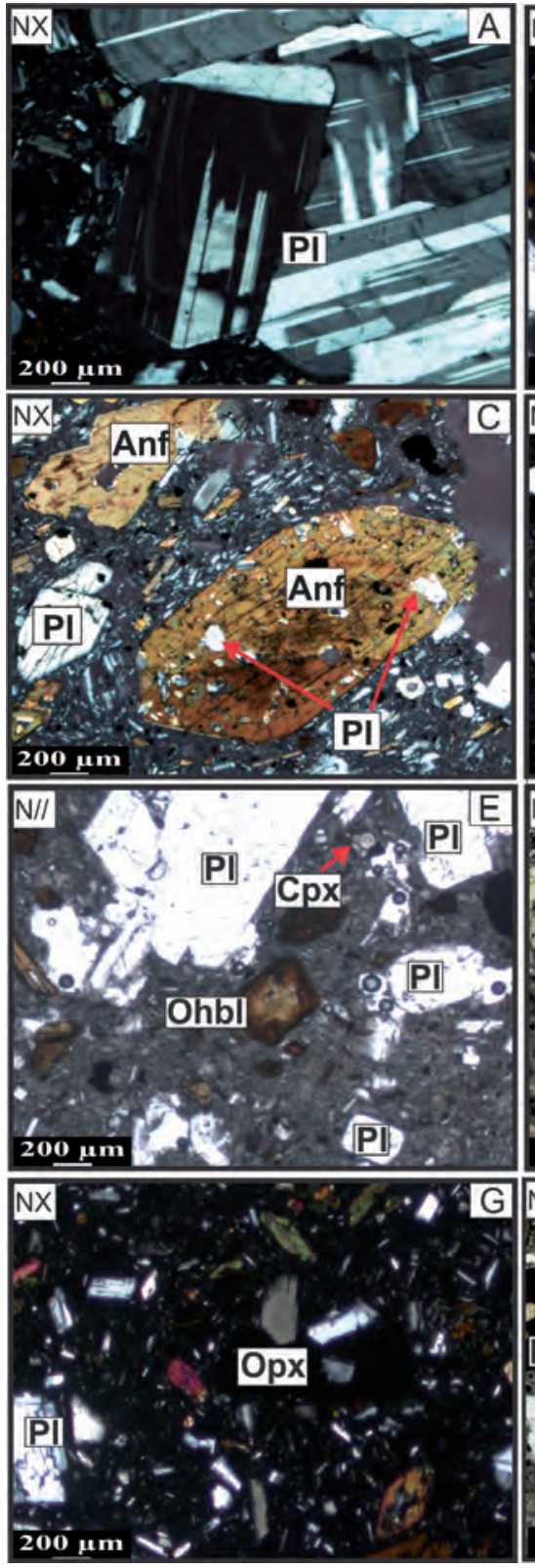

El piroxeno es la fase mineral que se presenta en menor abundancia (TABLA 1). Los cristales presentan formas predominantemente subhedrales y euhedrales con típicos hábitos prismáticos. Los cristales alcanzan tamaños hasta de 1,2 mm. Algunos minerales presentan alteraciones a epidota además de inclusiones de óxidos y microfenocristales de plagioclasa (FIGURA 2F). Tanto ortopiroxeno (FIGURA 2G) como clinopiroxeno (FIGURA 2H) son reconocidos y se encuentran en abundancias similares.
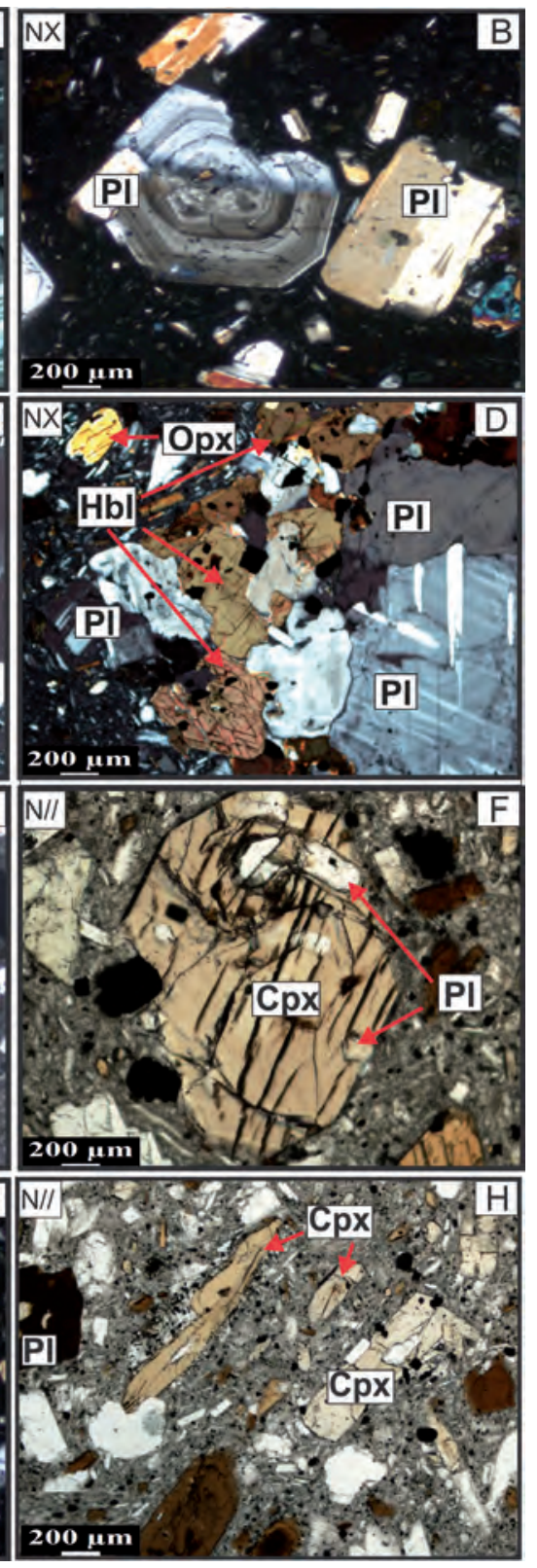

FIGURA 2. A. Maclas características de albita - periclina y textura glomeroporfirítica. B. A la izquierda plagioclasa zonada y a la derecha plagioclasa con macla de carlsbald. C. Inclusiones de plagioclasa dentro de cristal de hornblenda. D. Textura cumuloporfirítica. E. Oxihornblenda con pequeños bordes opacitizados. F. Cristal de clinopiroxeno con inclusiones de plagioclasa y óxidos. G. Ortopiroxeno. H. Clinopiroxeno. 


\section{Química mineral}

\section{Plagioclasa}

Un total de 139 análisis químicos fueron realizados en 72 cristales de plagioclasa (TABLA 2); 48 análisis en cristales homogéneos y 91 en 24 cristales zonados (MATERIAL SUPLEMENTARIO). Los resultados indican que los cristales medidos en todas las muestras tienen una composición que varía entre andesina y labradorita $\left(\mathrm{An}_{30}-\mathrm{An}_{70}\right)$ (FIGURA 3A) y que las zonaciones varían entre normales, inversas y oscilatorias (FIGURA 3B). Debido a que todas las muestras presentan la misma composición en plagioclasas, no se discriminan los resultados por muestra.

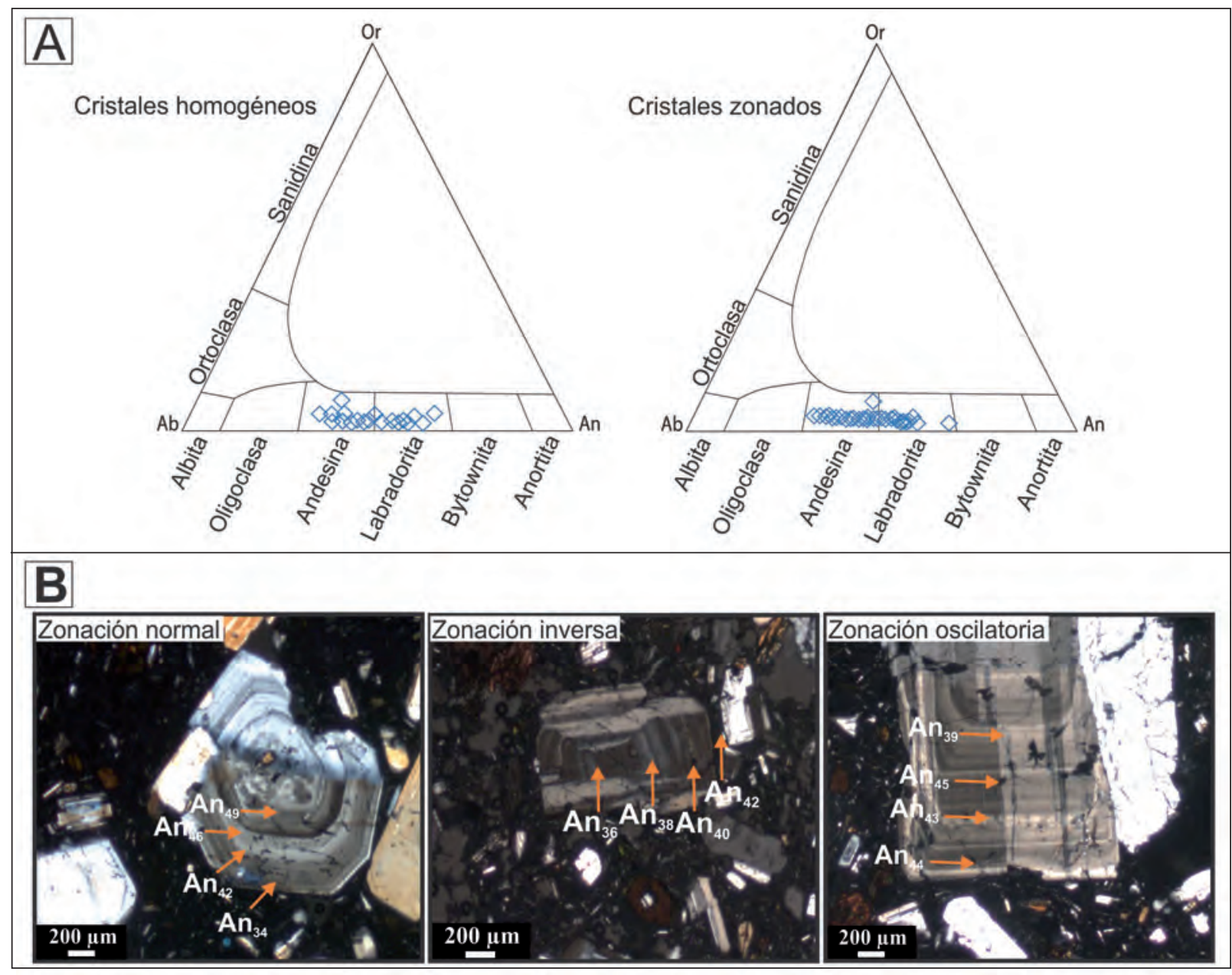

FIGURA 3. A. Diagramas ternarios de clasificación de feldespatos (Rahman y MacKenzie, 1969). B. Zonaciones en plagioclasa.

\section{Anfíbol}

Un total de 62 análisis químicos fueron realizados en diferentes cristales de anfíbol (TABLA 3; MATERIAL SUPLEMENTARIO). Los resultados muestran que los anfíboles son cálcicos (FIGURA 4) y que composicionalmente corresponden a magnesihornblenda, edenita y pargasita (FIGURA
5). Debido a que los cristales en todas las muestras presentan composiciones similares, no se hace distinción entre ellas en los resultados. Los anfíboles fueron clasificados usando los cationes por unidad de formula (c.p.f.u.) obtenidos del promedio de las cantidades máximas y mínimas de hierro férrico y ferroso presentes, a su vez obtenidas a partir de sus óxidos mayores (cf. Leake et al., 1997). 
TABLA 2. Óxidos mayores y cationes por unidad de fórmula para algunos cristales representativos de plagioclasa del domo del VCB.

\begin{tabular}{|c|c|c|c|c|c|c|c|c|c|c|}
\hline Muestra & CH1 & CH1 & CH2 & CH2 & $\mathrm{CH} 4$ & CH4 & CH6 & CH6 & CH7 & CH8 \\
\hline Código & $\underset{\text { plag_a }}{\text { CH1_ }}$ & $\underset{\text { plag_g }}{\text { CH1_ }}$ & $\underset{\text { plag_a }}{\mathrm{CH} 2}$ & $\underset{\text { plag_d }}{\mathrm{CH} 2_{2}}$ & $\underset{\text { plag_d }}{\mathrm{CH} 4}$ & $\underset{\text { plag_g }}{\mathrm{CH} 4}$ & $\underset{\text { plag_d }}{\text { CH6_ }}$ & $\underset{\text { plag_j }}{\mathrm{CH}_{\mathbf{j}}}$ & $\underset{\text { plag_m }}{\text { CH7_ }}$ & $\underset{\text { plag_m }}{\mathrm{CH}}$ \\
\hline $\mathrm{SiO}_{2}$ & 55,94 & 59,12 & 59,14 & 57,45 & 54,67 & 56,63 & 59,63 & 54,83 & 57,01 & 56,52 \\
\hline $\mathrm{Al}_{2} \mathrm{O}_{3}$ & 28,09 & 25,57 & 25,97 & 27,47 & 29,77 & 28,54 & 25,74 & 28,98 & 28,23 & 27,03 \\
\hline $\mathrm{CaO}$ & 9,71 & 7,49 & 7,79 & 8,93 & 11,31 & 10,08 & 7,49 & 11,08 & 9,68 & 8,07 \\
\hline $\mathrm{TiO}_{2}$ & 0,03 & 0,03 & 0,02 & 0,03 & 0,02 & 0,04 & 0,03 & 0,03 & 0,03 & 0,03 \\
\hline $\mathrm{Na}_{2} \mathrm{O}$ & 5,40 & 6,71 & 6,86 & 5,91 & 4,94 & 5,38 & 6,87 & 4,99 & 5,76 & 5,86 \\
\hline $\mathrm{MgO}$ & 0,01 & 0,00 & 0,00 & 0,01 & 0,01 & 0,01 & 0,01 & 0,01 & 0,01 & 0,02 \\
\hline $\mathrm{K}_{2} \mathrm{O}$ & 0,26 & 0,40 & 0,43 & 0,29 & 0,18 & 0,23 & 0,43 & 0,21 & 0,27 & 0,26 \\
\hline $\mathrm{FeO}$ & 0,22 & 0,25 & 0,22 & 0,25 & 0,21 & 0,23 & 0,23 & 0,21 & 0,22 & 0,22 \\
\hline TOTAL & 99,66 & 99,57 & 100,43 & 100,32 & 101,11 & 101,13 & 100,43 & 100,33 & 101,21 & 98,00 \\
\hline$\% A n$ & 49,06 & 37,25 & 37,60 & 44,72 & 55,27 & 50,18 & 36,65 & 54,42 & 47,39 & 42,51 \\
\hline \multicolumn{11}{|c|}{ Cationes por unidad de formula con base en 8 oxígenos } \\
\hline $\mathrm{Si}$ & 2,52 & 2,65 & 2,63 & 2,56 & 2,44 & 2,51 & 2,65 & 2,46 & 2,53 & 2,58 \\
\hline $\mathrm{Ti}$ & 0,00 & 0,00 & 0,00 & 0,00 & 0,00 & 0,00 & 0,00 & 0,00 & 0,00 & 0,00 \\
\hline $\mathrm{Al}$ & 1,49 & 1,35 & 1,36 & 1,45 & 1,57 & 1,49 & 1,35 & 1,54 & 1,48 & 1,45 \\
\hline $\mathrm{Mg}$ & 0,00 & 0,00 & 0,00 & 0,00 & 0,00 & 0,00 & 0,00 & 0,00 & 0,00 & 0,00 \\
\hline $\mathrm{Fe}^{2+}$ & 0,01 & 0,01 & 0,01 & 0,01 & 0,01 & 0,01 & 0,01 & 0,01 & 0,01 & 0,01 \\
\hline $\mathrm{Ca}$ & 0,47 & 0,36 & 0,37 & 0,43 & 0,54 & 0,48 & 0,36 & 0,53 & 0,46 & 0,39 \\
\hline $\mathrm{Na}$ & 0,47 & 0,58 & 0,59 & 0,51 & 0,43 & 0,46 & 0,59 & 0,43 & 0,50 & 0,52 \\
\hline K & 0,01 & 0,02 & 0,02 & 0,02 & 0,01 & 0,01 & 0,02 & 0,01 & 0,02 & 0,02 \\
\hline sum & 4,98 & 4,98 & 4,99 & 4,97 & 4,99 & 49,75 & 4,98 & 4,99 & 4,98 & 4,96 \\
\hline
\end{tabular}

$\mathrm{An}=(\mathrm{Ca} /(\mathrm{Na}+\mathrm{K}+\mathrm{Ca})) * 100$ 
TABLA 3. Óxidos mayores y cationes por unidad de fórmula para algunos cristales representativos de anfíbol del domo del VCB.

\begin{tabular}{|c|c|c|c|c|c|c|c|c|c|c|}
\hline Muestra & CH2 & CH2 & CH2 & CH1 & CH2 & CH4 & CH1 & CH2 & CH4 & CH4 \\
\hline Código & $\underset{\text { horn_a }}{\mathrm{CH} 2 \_}$ & $\underset{\text { horn_i }}{\mathrm{CH} 2}$ & $\begin{array}{c}\mathrm{CH} 2 \_ \\
\text {horn_m }\end{array}$ & $\begin{array}{c}\text { CH1_ } \\
\text { horn_e }\end{array}$ & $\begin{array}{c}\mathrm{CH} 2 \_ \\
\text {horn_r }\end{array}$ & $\begin{array}{c}\text { CH4_ } \\
\text { horn_f }\end{array}$ & $\begin{array}{c}\text { CH1_- } \\
\text { horn_q }\end{array}$ & $\begin{array}{c}\text { CH2_- } \\
\text { horn_q }\end{array}$ & $\begin{array}{c}\text { CH4_ } \\
\text { horn_a }\end{array}$ & $\begin{array}{l}\text { CH4_. } \\
\text { horn_i }\end{array}$ \\
\hline Nombre & Mg-Hbl & Mg-Hbl & Mg-Hbl & Prg & Prg & Prg & Prg & Eden & Eden & Eden \\
\hline $\mathrm{SiO}_{2}$ & 47,39 & 47,88 & 47,5 & 42,14 & 43,61 & 44,37 & 44,54 & 46,57 & 46,03 & 45,93 \\
\hline $\mathrm{Al}_{2} \mathrm{O}_{3}$ & 8,03 & 7,76 & 8,74 & 12,96 & 11,95 & 10,82 & 11,65 & 9,37 & 9,29 & 9,42 \\
\hline $\mathrm{CaO}$ & 10,28 & 10,79 & 10,19 & 11,11 & 11,21 & 11,31 & 11,31 & 11,03 & 11,05 & 10,97 \\
\hline $\mathrm{TiO}_{2}$ & 1,01 & 1,48 & 1,22 & 2,75 & 2,37 & 2,6 & 1,87 & 1,82 & 2,01 & 1,67 \\
\hline $\mathrm{Na}_{2} \mathrm{O}$ & 1,57 & 1,6 & 1,61 & 2,23 & 2,41 & 2,48 & 2,26 & 1,87 & 1,74 & 1,84 \\
\hline $\mathrm{MgO}$ & 14,2 & 14,62 & 14,07 & 14,19 & 14,9 & 16,14 & 15,43 & 13,72 & 13,63 & 13,81 \\
\hline $\mathrm{K}_{2} \mathrm{O}$ & 0,37 & 0,36 & 0,37 & 0,38 & 0,39 & 0,42 & 0,36 & 0,52 & 0,56 & 0,5 \\
\hline $\mathrm{P}_{2} \mathrm{O}_{5}$ & 0 & 0,01 & 0,05 & 0,02 & 0,01 & 0,03 & 0,03 & 0,02 & 0,01 & 0,03 \\
\hline $\mathrm{FeO}$ & 13,77 & 13,3 & 13,71 & 11,69 & 10,82 & 9,12 & 10,29 & 13,63 & 13,66 & 13,76 \\
\hline $\mathrm{MnO}$ & 0,45 & 0,29 & 0,39 & 0,12 & 0,11 & 0,1 & 0,09 & 0,26 & 0,25 & 0,28 \\
\hline TOTAL & 97,06 & 98,09 & 97,83 & 97,6 & 97,77 & 97,39 & 97,84 & 98,81 & 98,22 & 98,2 \\
\hline
\end{tabular}

Formula con base en 13 cationes

\begin{tabular}{|c|c|c|c|c|c|c|c|c|c|c|}
\hline${ }^{\mathrm{T}} \mathrm{Si}$ & 6,97 & 6,96 & 6,92 & 6,18 & 6,35 & 6,44 & 6,45 & 6,76 & 6,73 & 6,72 \\
\hline${ }^{\mathrm{T}} \mathrm{Al}{ }^{\mathrm{IV}}$ & 1,03 & 1,04 & 1,08 & 1,82 & 1,65 & 1,56 & 1,55 & 1,24 & 1,27 & 1,28 \\
\hline${ }^{\mathrm{T}} \mathrm{Al}{ }^{\mathrm{VI}}$ & 0,36 & 0,29 & 0,42 & 0,43 & 0,41 & 0,29 & 0,44 & 0,36 & 0,33 & 0,34 \\
\hline${ }^{\mathrm{C}} \mathrm{Ti}$ & 0,11 & 0,16 & 0,13 & 0,30 & 0,26 & 0,28 & 0,20 & 0,20 & 0,22 & 0,18 \\
\hline${ }^{\mathrm{C}} \mathrm{Cr}$ & 0,00 & 0,00 & 0,00 & 0,00 & 0,00 & 0,00 & 0,00 & 0,00 & 0,00 & 0,00 \\
\hline${ }^{\mathrm{C}} \mathrm{Fe}^{3+}$ & 0,00 & 0,00 & 0,00 & 0,00 & 0,00 & 0,00 & 0,00 & 0,00 & 0,00 & 0,00 \\
\hline${ }^{\mathrm{C}} \mathrm{Mg}$ & 3,11 & 3,17 & 3,06 & 3,11 & 3,24 & 3,49 & 3,33 & 2,97 & 2,97 & 3,01 \\
\hline${ }^{\mathrm{B}} \mathrm{Fe}^{2+}$ & 0,28 & 0,24 & 0,28 & 0,27 & 0,22 & 0,18 & 0,22 & 0,18 & 0,19 & 0,22 \\
\hline${ }^{\mathrm{C}} \mathrm{Fe}^{2+}$ & 1,41 & 1,38 & 1,39 & 1,17 & 1,10 & 0,93 & 1,02 & 1,48 & 1,48 & 1,47 \\
\hline${ }^{\mathrm{B}} \mathrm{Mn}$ & 0,06 & 0,04 & 0,05 & 0,02 & 0,01 & 0,01 & 0,01 & 0,03 & 0,03 & 0,03 \\
\hline${ }^{\mathrm{C}} \mathrm{Mn}$ & 0,00 & 0,00 & 0,00 & 0,00 & 0,00 & 0,00 & 0,00 & 0,00 & 0,00 & 0,00 \\
\hline${ }^{\mathrm{B}} \mathrm{Ca}$ & 1,62 & 1,68 & 1,59 & 1,75 & 1,75 & 1,76 & 1,75 & 1,72 & 1,73 & 1,72 \\
\hline${ }^{\mathrm{A}} \mathrm{Na}$ & 0,41 & 0,40 & 0,37 & 0,63 & 0,66 & 0,65 & 0,62 & 0,45 & 0,44 & 0,49 \\
\hline${ }^{\mathrm{B}} \mathrm{Na}$ & 0,04 & 0,05 & 0,08 & 0,00 & 0,02 & 0,05 & 0,01 & 0,07 & 0,05 & 0,03 \\
\hline${ }^{\mathrm{A}} \mathrm{K}$ & 0,07 & 0,07 & 0,07 & 0,07 & 0,07 & 0,08 & 0,07 & 0,10 & 0,10 & 0,09 \\
\hline
\end{tabular}

Mg-Hbl: Magnesihornblenda; Prg: Pargastia; Eden: Edenita. 


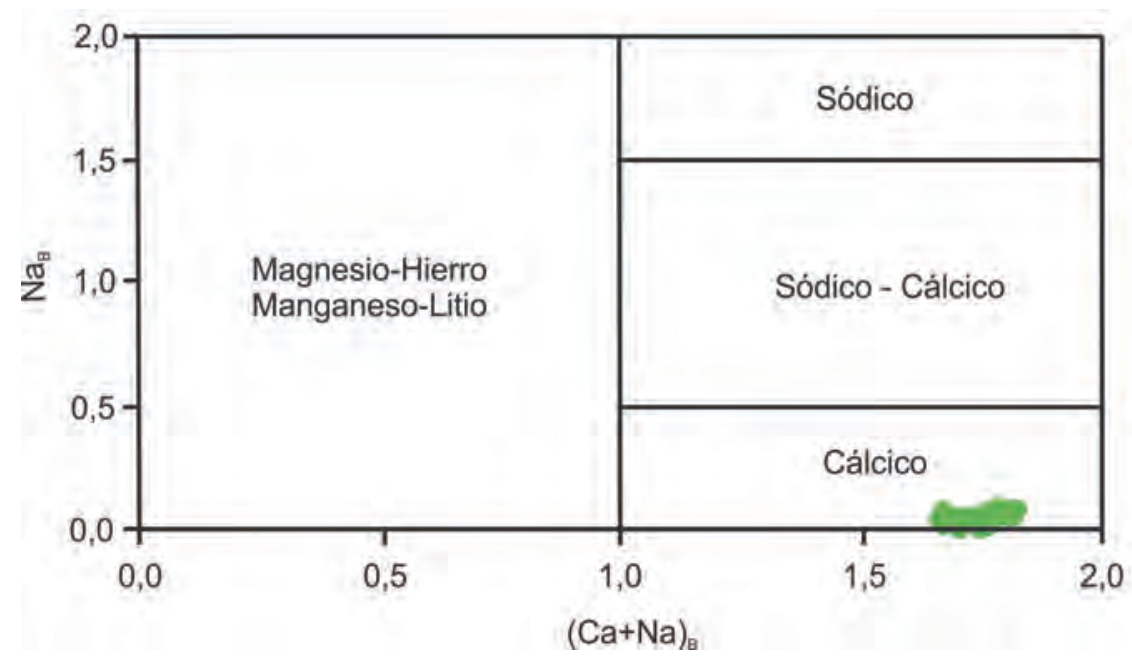

FIGURA 4. Clasificación del anfíbol con base en los valores de $(\mathrm{Ca}+\mathrm{Na})_{\mathrm{B}} v s \mathrm{Na}_{\mathrm{B}}$ (Leake et al., 1997). Todas las muestras se encuentran dentro del campo de anfíboles cálcicos y por ende no fueron discriminadas. $\mathrm{Na}_{\mathrm{B}} \mathrm{y} \mathrm{Ca}_{\mathrm{B}}$ hace referencia a c.p.f.u. en la posición B.
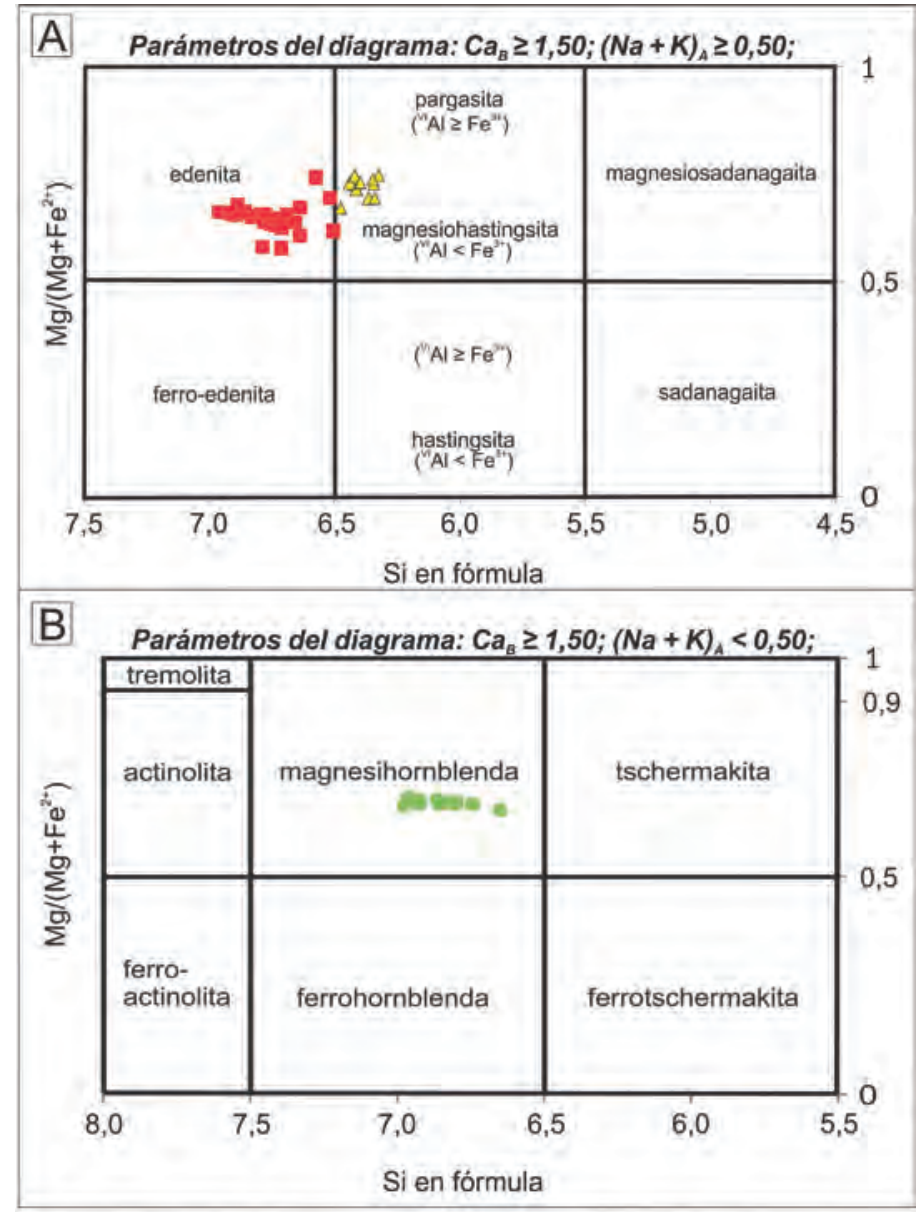

FIGURA 5. A. Diagrama binario de clasificación de anfíbol para condiciones de $(\mathrm{Na}+\mathrm{K})_{\mathrm{A}} \geq 0,50$; (Leake et al., 1997). B. Diagrama binario de clasificación de anfíbol para condiciones de $(\mathrm{Na}+\mathrm{K})_{\mathrm{A}}<0,50$; (Leake et al., 1997). $\mathrm{Ca}_{\mathrm{A}}, \mathrm{Na}_{\mathrm{A}}$ y $\mathrm{K}_{\mathrm{A}}$ hacen referencia a c.p.f.u. en la posición $\mathrm{A}$; $\mathrm{Ca}_{B}$ hace referencia a c.p.f.u. en la posición B. Los resultados no se presentan de manera discriminada, debido a que no existe variación en los tipos de anfíbol por muestra; los símbolos simplemente indican el tipo de anfíbol, según el mismo diagrama. 


\section{Piroxeno}

Un total de 35 análisis químicos se llevaron a cabo en diferentes cristales de piroxeno (TABLA 4; MATERIAL SUPLEMENTARIO). Los resultados en todas las muestras, indican que existe tanto enstatita (ortopiroxeno; $\mathrm{Wo}_{0-4}, \mathrm{En}_{64-70}, \mathrm{Fs}_{30-36}$ ), como augita (clinopiroxeno; $\mathrm{Wo}_{41-44}, \quad \mathrm{En}_{45-50}, \mathrm{Fs}_{10-12}$ ) (FIGURA $6 \mathrm{~A})$. Estos cristales caen en el campo de alta y media presión de formación (FIGURA 6B).

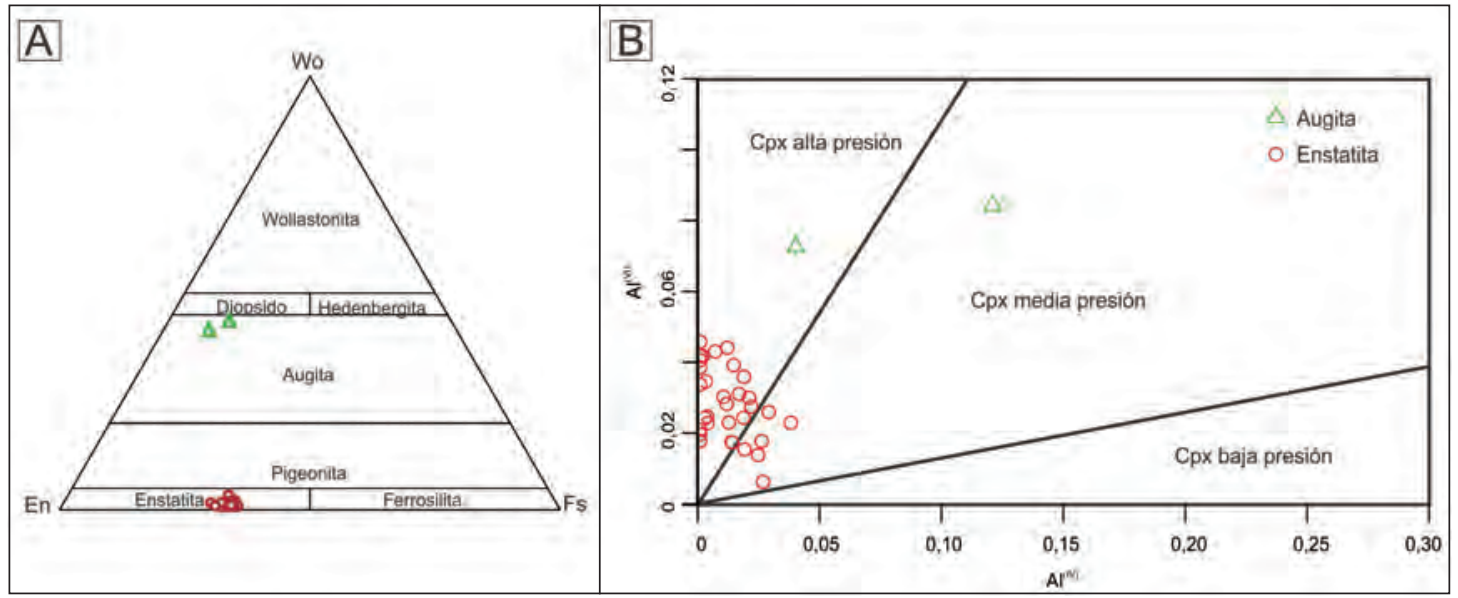

FIGURA 6. A. Diagrama ternario de clasificación de piroxeno (Morimoto, 1989). B. Diagrama binario de clasificación de piroxeno según la presión (Aoki y Shiba, 1973). Los resultados no se presentan de manera discriminada, debido a que no existe variación en los tipos de piroxeno por muestra; los símbolos simplemente indican ortopiroxeno o clinopiroxeno.

TABLA 4. Óxidos mayores y cationes por unidad de fórmula para algunos cristales representativos de piroxeno del domo del VCB.

\begin{tabular}{|c|c|c|c|c|c|c|c|c|c|c|}
\hline $\begin{array}{c}\text { Muestra } \\
\text { Código } \\
\text { Nombre }\end{array}$ & $\begin{array}{c}\text { CH2 } \\
\text { CH2_- } \\
\text { px_f } \\
\text { augita }\end{array}$ & $\begin{array}{c}\text { CH4 } \\
\text { CH4_- } \\
\text { px_d } \\
\text { augita }\end{array}$ & $\begin{array}{c}\text { CH2 } \\
\text { CH2_- } \\
\text { px_c } \\
\text { enstatita }\end{array}$ & $\begin{array}{c}\text { CH2 } \\
\text { CH2_- } \\
\text { px_e } \\
\text { enstatita }\end{array}$ & $\begin{array}{c}\text { CH2 } \\
\text { CH2_- } \\
\text { px_g } \\
\text { enstatita }\end{array}$ & $\begin{array}{c}\text { CH2 } \\
\text { CH2_- } \\
\text { px_i } \\
\text { enstatita }\end{array}$ & $\begin{array}{c}\text { CH4 } \\
\text { CH4_- } \\
\text { px_a } \\
\text { enstatita }\end{array}$ & $\begin{array}{c}\text { CH4 } \\
\text { CH4_ } \\
\text { px_c } \\
\text { enstatita }\end{array}$ & $\begin{array}{c}\text { CH4 } \\
\text { CH4_- } \\
\text { px_e } \\
\text { enstatita }\end{array}$ & $\begin{array}{c}\text { CH4 } \\
\text { CH4_- } \\
\text { px_g } \\
\text { enstatita }\end{array}$ \\
\hline $\mathrm{SiO}_{2}$ & 54,05 & 51,30 & 52,94 & 53,57 & 53,76 & 53,78 & 54,33 & 53,90 & 53,43 & 53,44 \\
\hline $\mathrm{Al}_{2} \mathrm{O}_{3}$ & 2,62 & 4,74 & 1,40 & 1,27 & 1,14 & 1,24 & 0,93 & 0,80 & 0,72 & 1,01 \\
\hline $\mathrm{CaO}$ & 20,06 & 20,44 & 0,40 & 0,46 & 0,40 & 0,41 & 0,40 & 0,72 & 0,48 & 0,72 \\
\hline $\mathrm{TiO}_{2}$ & 0,31 & 1,32 & 0,10 & 0,09 & 0,11 & 0,11 & 0,08 & 0,12 & 0,10 & 0,12 \\
\hline $\mathrm{Na}_{2} \mathrm{O}$ & 0,34 & 0,55 & 0,02 & 0,10 & 0,10 & 0,12 & 0,04 & 0,08 & 0,03 & 0,06 \\
\hline $\mathrm{MgO}$ & 17,18 & 14,84 & 22,63 & 22,77 & 22,80 & 22,50 & 22,77 & 23,37 & 22,96 & 22,77 \\
\hline $\mathrm{K}_{2} \mathrm{O}$ & 0,01 & 0,01 & 0,01 & 0,02 & 0,01 & 0,04 & 0,00 & 0,01 & 0,00 & 0,01 \\
\hline $\mathrm{FeO}$ & 5,77 & 7,48 & 22,14 & 21,83 & 21,57 & 21,37 & 21,94 & 20,93 & 21,14 & 21,66 \\
\hline $\mathrm{MnO}$ & 0,01 & 0,03 & 0,83 & 0,90 & 0,96 & 0,74 & 0,92 & 0,80 & 0,83 & 0,84 \\
\hline $\mathrm{P}_{2} \mathrm{O}_{5}$ & 0,17 & 0,17 & 0,00 & 0,00 & 0,00 & 0,00 & 0,00 & 0,00 & 0,00 & 0,00 \\
\hline TOTAL & 100,52 & 100,89 & 100,46 & 101,00 & 100,84 & 100,30 & 101,41 & 100,73 & 99,70 & 100,63 \\
\hline Mg\# & 84,12 & 78,83 & 57,37 & 57,54 & 57,49 & 57,07 & 56,83 & 58,74 & 57,98 & 57,74 \\
\hline
\end{tabular}

Cationes por unidad de formula con base en 6 oxígenos

\begin{tabular}{cllllllllll}
$\mathrm{Si}$ & 1,960 & 1,879 & 1,962 & 1,971 & 1,978 & 1,985 & 1,988 & 1,981 & 1,986 & 1,974 \\
$\mathrm{Ti}$ & 0,008 & 0,036 & 0,003 & 0,002 & 0,003 & 0,003 & 0,002 & 0,003 & 0,003 & 0,003 \\
$\mathrm{Al} \mathrm{I}^{\mathrm{IV}} \mathrm{r}$ & 0,040 & 0,121 & 0,038 & 0,029 & 0,022 & 0,015 & 0,012 & 0,019 & 0,014 & 0,026 \\
$\mathrm{Al}^{\mathrm{VII}}$ & 0,072 & 0,084 & 0,023 & 0,026 & 0,027 & 0,039 & 0,028 & 0,015 & 0,017 & 0,018 \\
$\mathrm{Al}^{\text {total }}$ & 0,112 & 0,205 & 0,061 & 0,055 & 0,049 & 0,054 & 0,040 & 0,035 & 0,032 & 0,044 \\
$\mathrm{Fe}$ & 0,175 & 0,229 & 0,686 & 0,672 & 0,664 & 0,660 & 0,671 & 0,643 & 0,657 & 0,669 \\
$\mathrm{Mn}$ & 0,000 & 0,001 & 0,026 & 0,028 & 0,030 & 0,023 & 0,029 & 0,025 & 0,026 & 0,026 \\
$\mathrm{Mg}$ & 0,929 & 0,810 & 1,250 & 1,249 & 1,251 & 1,238 & 1,242 & 1,280 & 1,272 & 1,254 \\
$\mathrm{Ca}$ & 0,779 & 0,802 & 0,016 & 0,018 & 0,016 & 0,016 & 0,016 & 0,028 & 0,019 & 0,028 \\
$\mathrm{Na}$ & 0,024 & 0,039 & 0,001 & 0,007 & 0,007 & 0,009 & 0,003 & 0,006 & 0,002 & 0,004 \\
$\mathrm{~K}$ & 0,000 & 0,001 & 0,000 & 0,001 & 0,000 & 0,002 & 0,000 & 0,000 & 0,000 & 0,000 \\
Suma & 3,988 & 4,002 & 4,006 & 4,003 & 3,998 & 3,990 & 3,991 & 4,002 & 3,997 & 4,003 \\
\hline
\end{tabular}




\section{DISCUSIÓN}

\section{Geotermobarometría}

El uso de geotermobarómetros a partir de análisis químicos de óxidos mayores en minerales permite determinar condiciones de presión y temperatura a las cuales ocurrió la cristalización de las fases analizadas y, por ende, las condiciones del magma en algún momento de su recorrido hacia la superficie (e.g. Putirka, 2008). Usualmente, los geotermobarómetros, requieren la relación entre la composición del vidrio o de la roca total y la composición de un mineral; no obstante, también es posible utilizar exclusivamente la composición de determinadas fases minerales. Así, en este estudio se aplican termobarómetros de anfíbol y piroxeno que usan solamente química mineral. Estos valores son usados para conocer tanto las profundidades a las que se formaron los minerales, como las condiciones de fugacidad de oxígeno y de contenido de agua dentro del sistema.

\section{Anfíbol}

Aplicando análisis de geotermobarometría de anfíbol (Ridolfi et al., 2010), fue posible diferenciar dos poblaciones de acuerdo a sus condiciones de temperatura, presión, fugacidad de oxígeno y contenido de agua (FIGURA 7): la población (1) definida por anfíbol tipo pargasita, y la población (2) definida por anfíbol tipo edenita y magnesihornblenda.

Vale la pena aclarar que los minerales de oxihornblenda no fueron utilizados para estos cálculos ya que para la aplicación de este geotermobarómetro los cristales no deben presentar evidencias de desequilibrio, como por ejemplo texturas de alteraciones o reabsorción (Ridolf et al., 2010). Tales condiciones se describen a continuación:

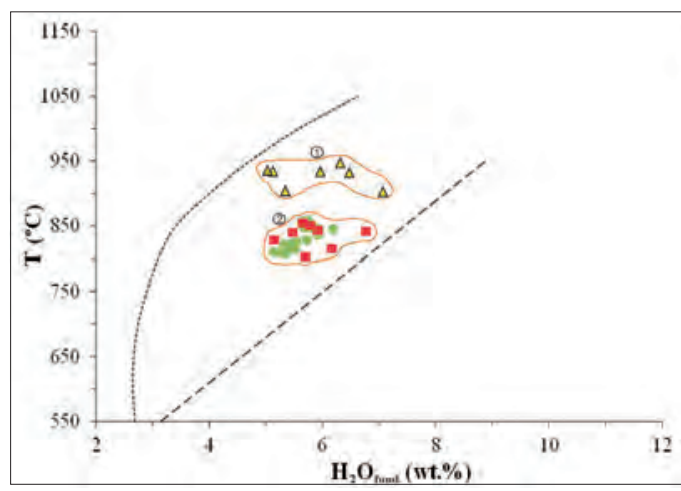

A
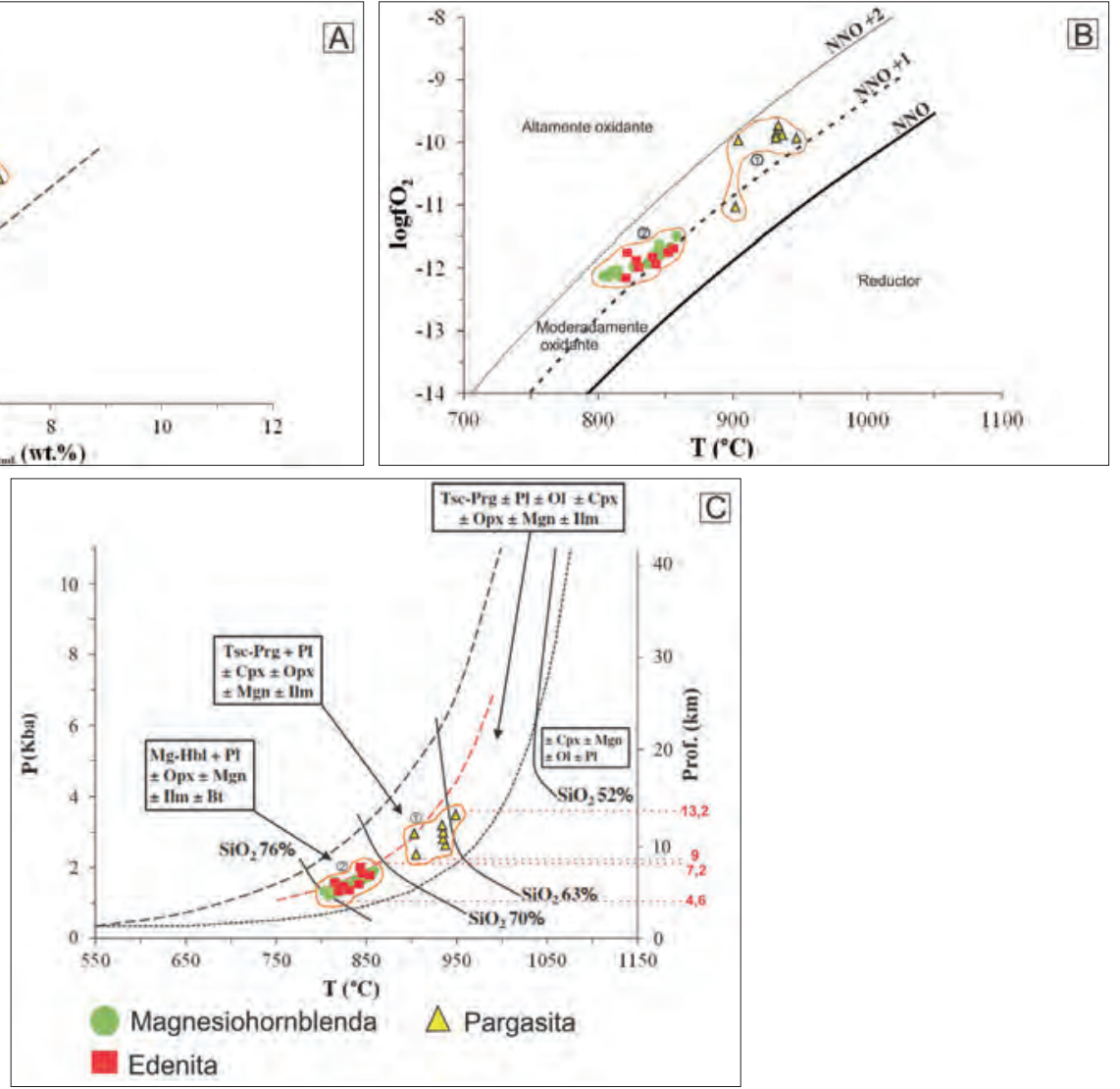

FIGURA 7. A. Diagrama de temperatura (T) vs agua en el fundido $\left(\mathrm{H}_{2} \mathrm{O}\right.$ fund.) para anfíboles cálcicos (Ridolfi et al., 2010). Las dos poblaciones se encuentran encerradas por una línea de color naranja y corresponden a los cristales con similares condiciones en cuanto a contenido de agua en el fundido ( $\mathrm{H}_{2} \mathrm{O}$ fund. wt\%) y temperatura (T). B. Diagrama de fugacidad de oxígeno $\left(\operatorname{logfO}_{2}\right)$ vs temperatura (T) para anfíboles cálcicos (Ridolfi et al., 2010). Las dos poblaciones se encuentran encerradas por una línea de color naranja y corresponden a los cristales que se encuentran en un mismo rango de temperatura $(\mathrm{T})$ y fugacidad de oxígeno $\left(\operatorname{logf\mathrm {O}_{2}}\right)$. La ubicación de las poblaciones dentro de las curvas niquel - óxido de niquel (NNO) y NNO+2 muestran que los anfíboles cálcicos se encuentran dentro de condiciones moderadamente oxidantes. C. Diagrama de presión (P) vs temperatura (T) para anfíboles cálcicos (Ridolfi et al., 2010). Las dos poblaciones se encuentran encerradas por una línea de color naranja y corresponden a los cristales que se encuentran en un mismo rango de presión (P) y temperatura (T). Los cuadros indican las paragénesis minerales asociadas con los porcentajes en peso de $\mathrm{SiO}_{2}$ de la roca. 


\section{Temperatura, presión y profundidad}

Los valores arrojados por el geotermobarómetro de Ridolfi et al. (2010) muestran que los cristales de anfíbol se formaron en rangos de temperatura que varían desde $804 \pm 22^{\circ} \mathrm{C}$ hasta $948 \pm 22^{\circ} \mathrm{C}$ y presiones entre $1,2 \mathrm{y}$ 3,5 kba (FIGURA 7; TABLA 5). Específicamente la población (1) presenta temperaturas desde $902 \pm 22^{\circ} \mathrm{C}$ hasta $948 \pm 22^{\circ} \mathrm{C}$ y presiones entre 2,3 y 3,5 kba, mientras que la población (2) presenta temperaturas desde $804 \pm 22^{\circ} \mathrm{C}$ hasta $858 \pm 22^{\circ} \mathrm{C}$ y presiones entre 1,0 y 2,0 kba (FIGURA 7). Si se asume un peso específico de la corteza de $2,70 \mathrm{~g} / \mathrm{cm}^{3}$ (Ridolfi et al., 2010), es posible inferir con los resultados obtenidos para cada población de anfíbol, que estas dos poblaciones cristalizaron desde 13,2 hasta 4,6 km de profundidad (Ridolfi et al., 2010). Esto concuerda con los valores máximos esperados para magmas andesíticos, donde el anfíbol es inestable a temperaturas superiores a $\sim 950^{\circ} \mathrm{C}$ y presiones inferiores a los 0,5 kba ( 2 km de profundidad) (Maksimov, 2009).

TABLA 5. Valores de T, $\mathrm{P}$, profundidad, $\operatorname{logfO}_{2}$ y porcentaje en peso de $\mathrm{H}_{2} \mathrm{O}$ en el fundido obtenidos a partir del análisis químico de elementos mayores de minerales de anfíbol del domo intracratérico del VCB. Valores obtenidos usando el geotermobarómetro de Ridolfi et al. (2010).

\begin{tabular}{|c|c|c|c|c|c|c|}
\hline & Muestra & $\mathrm{T}\left({ }^{\circ} \mathrm{C}\right)$ & P (MPa) & Profundidad (km) & $\operatorname{logfO}$ & Hofund.(wt.\%) \\
\hline \multirow{16}{*}{ 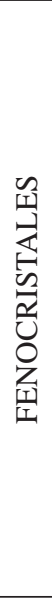 } & CH1_horn_a & 829 & 152 & 5,8 & $-11,9^{2}$ & 5,7 \\
\hline & CH1_horn_e & 973 & 450 & 17,0 & $-9,7$ & 7,0 \\
\hline & CH1_horn_q & 932 & 319 & 12,0 & $-9,9$ & 6,5 \\
\hline & CH2_horn_a & 804 & 135 & 5,1 & $-12,1$ & 5,7 \\
\hline & CH2_horn_i & 810 & 125 & 4,7 & $-12,1$ & 5,3 \\
\hline & CH2_horn_m & 815 & 157 & 5,9 & $-12,0$ & 6,2 \\
\hline & CH2_horn_q & 852 & 185 & 7,0 & $-11,7$ & 5,8 \\
\hline & CH4_horn_a & 856 & 185 & 7,0 & $-11,7$ & 5,6 \\
\hline & CH4_horn_i & 858 & 190 & 7,2 & $-11,5$ & 5,7 \\
\hline & CH4_horn_q & 822 & 141 & 5,3 & $-11,7$ & 5,3 \\
\hline & CH4_horn_m & 829 & 140 & 5,3 & $-12,0$ & 5,1 \\
\hline & CH5_horn_m & 841 & 155 & 5,9 & $-11,8$ & 5,5 \\
\hline & CH6_horn_a & 853 & 178 & 6,7 & $-11,7$ & 5,7 \\
\hline & CH6_horn_i & 837 & 162 & 6,1 & $-11,9$ & 5,9 \\
\hline & CH6_horn_e & 845 & 181 & 6,8 & $-11,6$ & 6,2 \\
\hline & CH6_horn_q & 822 & 137 & 5,2 & $-12,2$ & 5,5 \\
\hline \multirow{15}{*}{ 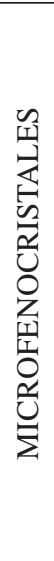 } & CH1_horn_f & 848 & 169 & 6,4 & $-11,8$ & 5,7 \\
\hline & CH2_horn_b & 904 & 235 & 8,9 & $-10,0$ & 5,4 \\
\hline & CH2_horn_f & 842 & 201 & 7,6 & $-11,9$ & 6,8 \\
\hline & CH2_horn_j & 902 & 296 & 11,2 & $-11,0$ & 7,1 \\
\hline & CH2_horn_r & 948 & 349 & 13,2 & $-9,9$ & 6,3 \\
\hline & CH4_horn_f & 937 & 265 & 10,0 & $-9,9$ & 5,0 \\
\hline & CH4_horn_j & 810 & 125 & 4,7 & $-12,1$ & 5,1 \\
\hline & CH4_horn_r & 934 & 282 & 10,6 & $-9,8$ & 5,1 \\
\hline & CH5_horn_b & 934 & 300 & 11,3 & $-9,8$ & 6,0 \\
\hline & CH5_horn_f & 815 & 130 & 4,9 & $-12,1$ & 5,5 \\
\hline & CH5_horn_j & 808 & 122 & 4,6 & $-12,1$ & 5,3 \\
\hline & CH5_horn_m & 826 & 147 & 5,5 & $-12,0$ & 5,5 \\
\hline & CH5_horn_r & 845 & 165 & 6,2 & $-11,7$ & 5,9 \\
\hline & CH6_horn_f & 844 & 171 & 6,5 & $-11,8$ & 5,9 \\
\hline & CH6_horn_n & 814 & 132 & 5,0 & $-12,1$ & 5,4 \\
\hline
\end{tabular}

Porcentaje de agua en el fundido $\left(\mathrm{H}_{2} \underline{\mathrm{O}}\right)$

Para el domo del VCB, los valores deducidos para el contenido de $\mathrm{H}_{2} \mathrm{O}$ en el fundido no muestran una relación directa con la composición mineralógica de las dos poblaciones de anfíbol, ni con las temperaturas y presiones de cristalización para estos grupos. De hecho, las cantidades de $\mathrm{H}_{2} \mathrm{O}$ son similares en ambas etapas de cristalización (FIGURA 7A). La población (1) fue formada con contenidos de $\mathrm{H}_{2} \mathrm{O}$ en el fundido entre 5,0 y 7,1 wt.\%, mientras que la población (2) con contenidos más restringidos que varían entre 5,3 y 6,8 wt.\% (FIGURA 7C). Esto sugiere que el contenido de $\mathrm{H}_{2} \mathrm{O}$ dentro de la masa fundida es independiente a la formación del anfíbol dentro del sistema (Eggler, 1972; Eggler y Burnham, 1973), y que la cristalización del anfíbol no solo dependió de los contenidos de $\mathrm{H}_{2} \mathrm{O}$ en el fundido, sino también de la presión total en el sistema, la composición de la fase fluida, la temperatura y las 
condiciones redox en la cámara magmática (Maksimov, 2009). Para el domo del VCB, los geotermobarómetros indican que la cristalización de las dos poblaciones de anfíbol ocurrió a una presión superior a 1 kba (Maksimov, 2009). Adicionalmente, la abundancia de anfíbol y la relativa escasez de piroxeno sugieren altos contenidos de agua en el sistema (entre 4 y 5 wt.\%) (Lescinsky, 1990).

\section{Fugacidad del oxígeno ( $\mathrm{fO}_{2}$ )}

Para el VCB, los valores de $\mathrm{fO}_{2}$ asociados con el fundido evidencian que el magma tuvo condiciones moderadamente oxidantes durante la formación de los cristales (FIGURA 7B) (O’Neill y Pownceby, 1993; Martel et al., 1999). Los valores calculados para el VCB varían entre -11,0 y -9,8 (NNO) para la población (1) y -12,2 y -11,5 (NNO) para la población (2) (FIGURA 7B). Ambos rangos de valores sugieren que los minerales definidos como óxidos tuvieron condiciones redox aptas para su formación y que éstos pueden corresponder a magnetita, titanomagnetita e ilmenita (Martel et al., 1999); entre los reguladores $\mathrm{NNO}$ a $\mathrm{NNO}+2$, la magnetita, la plagioclasa y en ocasiones la titanomagnetita, pueden coexistir dentro de un fundido de composición andesítica (Sekine et al., 1979). Adicionalmente, vale la pena destacar que entre los reguladores $\mathrm{NNO}$ y $\mathrm{NNO}+2$ se tiene la estabilidad del ortopiroxeno y que por encima del regulador $\mathrm{NNO}+2$ disminuye la formación de piroxeno cálcico (Rutherford et al., 1985; Rutherford y Devine, 1988; Martel et al., 1999). De esta manera se puede explicar la aparición tanto de clinopiroxeno como de ortopiroxeno en la paragénesis de las rocas analizadas, las cuales se formaron en condiciones moderadamente oxidantes entre NNO y NNO+2.

\section{$\underline{\text { Piroxeno }}$}

Aplicando análisis de geotermobarometría en dos piroxenos (ortopiroxeno y clinopiroxeno), fue posible obtener valores de temperatura (Ecuación 36; Putirka, 2008) y presión (Ecuación 38; Putirka, 2008) de $914 \pm 97^{\circ} \mathbf{C}$ y $18,5 \pm 9,2 \mathrm{kba}$, respectivamente. Para estos análisis, el coeficiente de equilibrio entre los dos piroxenos fue de $K_{D \text { Ens-Aug }}$ : 0,59 $\pm 0,07$, el cual se determinó usando todos los análisis químicos de ortopiroxeno en relación con los análisis químicos del clinopiroxeno CH4_px_d, este último debido a que su composición representa un magma menos evolucionado (TABLA 4). Al respecto, es importante mencionar que estos valores obtenidos son menores a los valores de $K_{D}$ sugeridos por Putirka (2008; hoja de cálculo en su página Web) como los valores que representan cristales en equilibrio $\left(K_{D}: 0,7 \pm 0,2\right)$, en condiciones subsolidas, por lo que las condiciones de presión y temperatura para la cristalización de piroxeno calculados en este trabajo, deben ser tomados con precaución. Análisis adicionales ayudarían a constreñir estos resultados. Por ejemplo, el diagrama $\mathrm{Al}^{\mathrm{VI}} / \mathrm{Al}^{\mathrm{IV}}$ propuesto por Aoki y Shiba (1973) (FIGURA 6B), indica que los cristales de piroxeno cristalizaron en condiciones medias y altas de presión, lo cual es concordante con los valores aquí obtenidos. No obstante, análisis químicos de roca total permitirían aplicar otros geotermobarómetros (e.g. Putirka, 2008) y por ende restringir los valores obtenidos.

\section{Evidencia de evolución magmática}

Las características texturales y químicas observadas en las rocas del domo del VCB permiten inferir diferentes procesos dentro de la cámara magmática. Por ejemplo, los cristales de plagioclasa mostraron zonaciones normales, inversas y oscilatorias que evidencian cambios progresivos, regresivos, o variables en la composición de calcio y sodio dentro de la estructura cristalina, indicando procesos de inestabilidad de los cristales durante la evolución magmática. Este mineral es fácilmente afectado por cambios en las condiciones magmáticas, ya sean cambios de presión, temperatura y/o procesos convectivos en la cámara (eg. Cashman, 1993; Berlo et al., 2007). Por lo tanto, las características observadas sugieren la ocurrencia de tres procesos principales. La zonación normal es el resultado de procesos de cristalización fraccionada, donde la cantidad de calcio disminuye en el fundido a medida que éste es incorporado en las primeras fases minerales que cristalizan (Vernon et al., 2004; Shcherbakov et al., 2011). Las zonaciones inversa y oscilatoria, al igual que texturas de tamiz y reabsorción, indican desequilibrio en las condiciones de cristalización, ya sea por cambios de temperatura, presión o composición (Cashman, 1993; Izbekov et al., 2002; Vernon et al., 2004; Shcherbakov et al., 2011). Dicho desequilibrio puede ocurrir por procesos convectivos en la cámara magmática (Singer et al., 1995; Ginibre y Wörner, 2007), o durante el ascenso del magma a superficie (Tsuchiyama, 1985; Vernon et al., 2004; Shcherbakov et al., 2011). De hecho, la presencia de oxihornblenda, también es evidencia de inestabilidad asociada a variaciones en la temperatura y descompresiones, relacionados con el ascenso del magma (Rutherford y Hill, 1993; Browne y Gardner, 2006).

\section{Modelo de cristalización de los minerales y la cámara magmática}

Las dos poblaciones de anfíbol identificadas en este trabajo sugieren que las rocas del domo del VCB son el resultado de dos fases de cristalización. La población 
de pargasita representa mayores temperaturas y presiones de cristalización en comparación con la población de edenita y magnesihornblenda. La presencia de dos poblaciones, claramente definidas por sus temperaturas de formación, indican que entre 858 y $902^{\circ} \mathrm{C}$ no hubo cristalización de anfíbol, lo que sugiere un tiempo de inestabilidad en el sistema que inhibió la cristalización de este mineral. Este hecho puede estar relacionado con un ascenso relativamente rápido del magma desde una zona definida entre 13,2 y $9 \mathrm{~km}$ hasta otra entre 7,2 y 4,6 km como lo indican los valores obtenidos. No obstante, más que dos zonas de almacenamiento asociadas con diferentes momentos de cristalización, se proponen dos periodos de formación en dos zonas con condiciones estables para ello dentro de una misma cámara magmática ubicada entre 13,2 y 4,6 km de profundidad (FIGURA 8). Los cristales de piroxeno con condiciones de temperatura y presión más altas indican condiciones de formación a mayor profundidad, mientras que los cristales de plagioclasa sugieren condiciones de formación tanto a mayor como a menor profundidad en relación con la cámara magmática mencionada (FIGURA 8). Evidencia de esto son los cristales de plagioclasa como inclusiones tanto en los cristales de piroxeno como de anfíbol, así como la diferencia en composición desde labradorita $\left(\mathrm{An}_{50-70}\right)$ hasta andesina $\left(\mathrm{An}_{30-50}\right)$ y la presencia de textura seriada y de microlitos que indican diferentes momentos de cristalización a lo largo del magma durante el ascenso a superficie. Al respecto, Londoño (2016) propone un reservorio magmático alimentando las diferentes cámaras magmáticas de los volcanes de la PVTSC a una profundidad entre 20 y 30 km (ver también Murcia et al., en prensa). De esta manera se propone una cámara magmática superficial para el VCB por encima del reservorio mencionado (FIGURA 8).

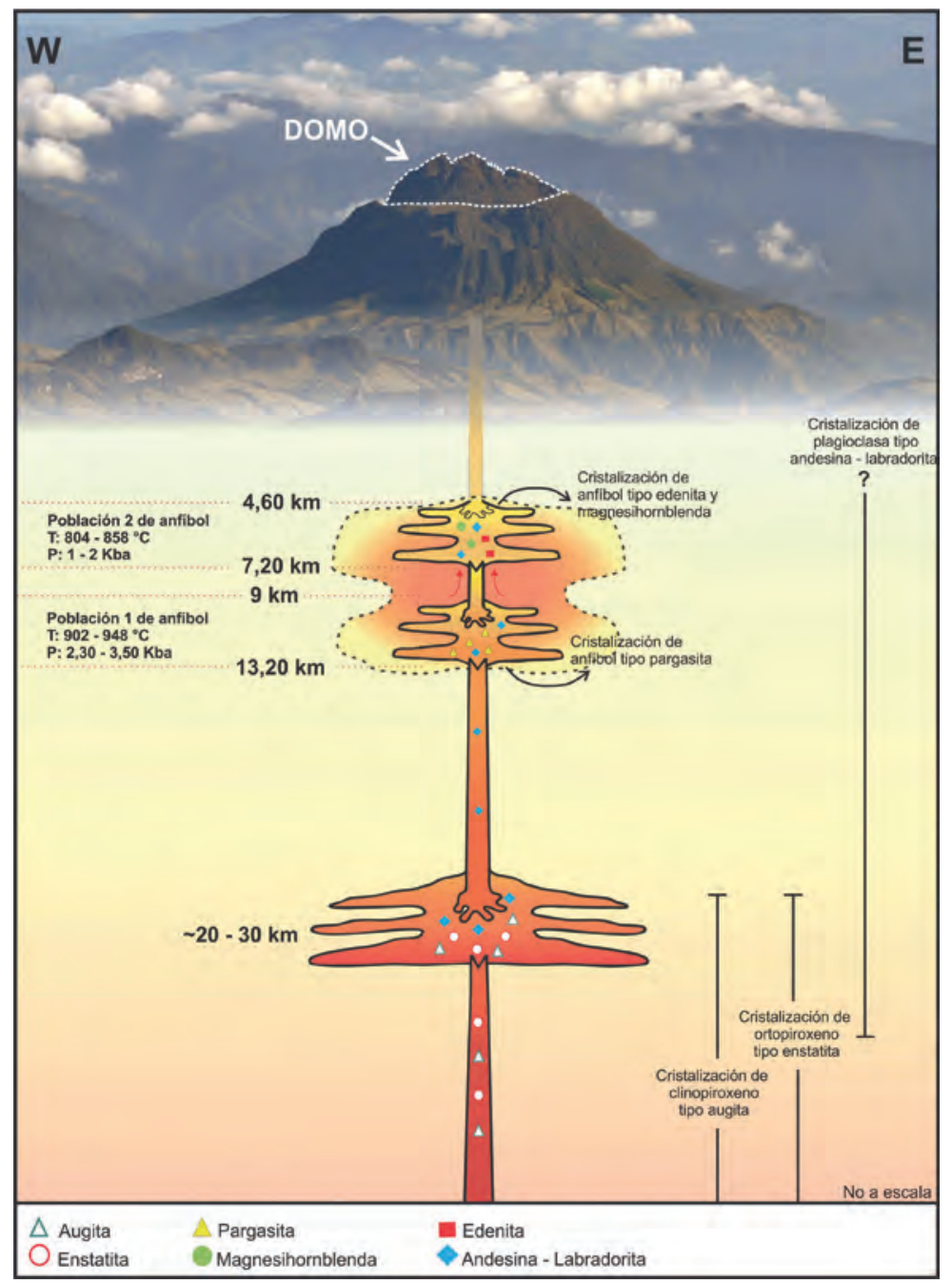

FIGURA 8. Modelo de evolución magmática para el domo que representa la última fase eruptiva de la última erupción del VCB. Fotografía del VCB tomada de www.sgc.gov.co. 


\section{CONCLUSIONES}

- Las fases minerales presentes en las rocas del domo del VCB son definidas como: plagioclasa tipo andesina - labradorita $\left(\mathrm{An}_{30-70}\right)$, hornblenda y oxihornblenda tipo edenita, pargasita y magnesihornblenda, y piroxeno de tipo augita y enstatita.

- La composición de enstatita y augita $\left(\mathrm{K}_{\mathrm{D} \text { Ens-Aug }}\right.$ : $0,59 \pm 0,07)$, sugieren que la cristalización de piroxeno ocurrió a $914 \pm 97^{\circ} \mathrm{C}$ y $18,5 \pm 9,2 \mathrm{kba}$.

- Zonaciones inversas y oscilatorias, al igual que texturas de tamiz y reabsorción principalmente en plagioclasa, al igual que la presencia de oxihornblenda, son evidencia de inestabilidad asociada a variaciones en la temperatura debido a descompresión relacionado con el ascenso del magma.

- Los cristales de anfíbol fueron clasificados como cálcicos y dos poblaciones fueron definidas: una con anfíbol tipo pargasita y otra con anfíbol tipo edenita y magnesihornblenda. Estas poblaciones corresponden a dos momentos de cristalización a dos profundidades diferentes dentro de una misma cámara magmática. La población de pargasita representa temperaturas de $902 \pm 22^{\circ} \mathrm{C}$ hasta $948 \pm 22^{\circ} \mathrm{C}$ y presiones entre 2,3 y 3,5 kba correspondientes a profundidades desde 13,2 hasta $9 \mathrm{~km}$. La población de edenita y magnesihornblenda representa temperaturas desde $804 \pm 22^{\circ} \mathrm{C}$ hasta $858 \pm 22^{\circ} \mathrm{C}$ y presiones entre $1 \mathrm{y}$ $2 \mathrm{kba}$, que corresponden a profundidad desde7,2 hasta $4,6 \mathrm{~km}$.

\section{AGRADECIMIENTOS}

Al personal del Instituto de Investigaciones en Estratigrafía (IIES), por su gran apoyo para el desarrollo de este proyecto y al Earth Observatory of Singapore, por su gestión para los análisis de química mineral. Finalmente, a dos revisores anónimos quienes ayudaron a mejorar el manuscrito.

\section{REFERENCIAS}

Acosta, J., Velandia, F., Osorio, J., Lonergan, L., and Mora, H. (2007). Strike-slip deformation within the Colombian Andes. Geological Society, London, Special Publications, 272, 303-319. doi: 10.1144/GSL.SP.2007.272.01.16.

Aoki, K.I., and Shiba, I. (1973). Pyroxenes from lherzolite inclusions of Itinome-gata,
Japan. Lithos, 6(1), 41-51. doi: 10.1016/00244937(73)90078-9.

Berlo, K., Blundy, J., Turner, S., and Hawkesworth, C. (2007). Textural and chemical variation in plagioclase phenocrysts from the 1980 eruptions of Mount St. Helens, USA. Contributions to Mineralogy and Petrology, 154(3), 291-308. doi: 10.1007/s00410-007-0194-8.

Blanco-Quintero, I.F., García-Casco, A., Toro, L.M., Moreno, M., Ruiz, E.C., Vinasco, C.J., Cardona, A., Lázaro, C., and Morata, D. (2014). Late Jurassic terrane collision in the northwestern margin of Gondwana (Cajamarca Complex, eastern flank of the Central Cordillera, Colombia). International Geology Review, 56(15), 1852-1872.

Borrero, C.A., and Naranjo, J.L. (1990). Casabianca Formation: A Colombian example of volcanisminduced aggradation in a fluvial basin. Journal of Volcanology and Geothermal Research, 41(1), 253-267. doi: 10.1016/0377-0273(90)90091-S.

Bourdon, E., Eissen, J., Gutscher, M., Monzier, M., Hall, M., and Cotten, J. (2003). Magmatic response to early aseismic ridge subduction: the Ecuadorian margin case (South America). Earth and Planetary Science Letters, 205(3-4), 123138. doi: 10.1016/S0012-821X(02)01024-5.

Browne, B., and Gardner, J. (2006). The influence of magma ascent path on the texture, mineralogy, and formation of hornblende reaction rims. Earth and Planetary Science Letters, 246(3-4), 161176. doi: 10.1016/j.epsl.2006.05.006.

Calvache, M., Williams, S.N., and Young, R.H. (1987). Distribution and volumes of deposits and dynamics of eruptions of Nevado del Ruiz and Cerro Bravo volcanoes, Colombia, over the past 2100 years (abs.). EOS Earth \& Space Science News, 67(16), 405.

Cárdenas, P. (2004). Modelo y cartografía estructural del sistema de fallas de Palestina en sector del Parque Natural de los Nevados. Tesis, Universidad de Caldas, Manizales, Colombia.

Cárdenas, P., Ocampo, P., Cocuy, C., Garcés, J., y Marín, L. (2004). Modelo y cartografía estructural del sistema de fallas de Palestina y Mulatos. INGEOMINAS, Bogotá. 
Cashman, K. (1993). Relationship between plagioclase crystallization and cooling rate in basaltic melts. Contributions to Mineralogy and Petrology, 113(1), 126-142. doi: 10.1007/BF00320836.

CHEC. (1983). Investigación geotérmica Macizo Volcánico del Ruiz. Informe Final. Central Hidroeléctrica de Caldas.

Cortés, J. (2015). CFU-PINGU. Consultado el 15 de junio de 2017. https://vhub.org/resources/ cfupingu.2015.

Eggler, D. (1972). Amphibole stability in $\mathrm{H}_{2} \mathrm{O}-$ undersaturated calc-alkaline melts. Earth and Planetary Science Letters, 15(1), 28-34. doi: 10.1016/0012-821X(72)90025-8.

Eggler, D., and Burnham, C. (1973). Crystallization and fractionation trends in the system andesite$\mathrm{H}_{2} \mathrm{O}-\mathrm{CO}_{2}-\mathrm{O}_{2}$ at pressures to $10 \mathrm{~Kb}$. Geological Society of America Bulletin, 84, 2517-2532.

Ginibre, C., and Wörner, G. (2007). Variable parent magmas and recharge regimes of the Parinacota magma system (N. Chile) revealed by Fe, Mg and Sr zoning in plagioclase. Lithos, 98(1-4), 118140. doi: 10.1016/j.lithos.2007.03.004.

González, L., y Jaramillo, C. (2002). Estudio neotectónico multidisciplinario aplicado a la falla Villamaría-Termales. Tesis, Departamento de Ciencias Geológicas, Universidad de Caldas, Manizales, Colombia.

Guzmán, J., Franco, G., Ochoa, M., Paris, G., y Taboada, A. (1998). Proyecto para la mitigación del riesgo sísmico de Pereira Dosquebradas y Santa Rosa de Cabal: Evaluación neotectónica. Informe Final, Corporación Autónoma Regional de Risaralda, Pereira, Colombia.

Izbekov, P., Eichelberger, J., Patino, L., Vogel, T., and Ivanov, B. (2002). Calcic cores of plagioclase phenocrysts in andesite from Karymsky volcano: Evidence for rapid introduction by basaltic replenishment. Geology, 30(9), 799-802. doi: 10.1130/0091-7613(2002)030<0799:CCOPPI>2. $0 . \mathrm{CO} ; 2$.

Janoušek, V., Farrow, C., and Erban, V. (2006). Interpretation of whole-rock geochemical data in igneous geochemistry: introducing Geochemical
Data Toolkit (GCDkit). Journal of Petrology, 47(6), 1255-1259. doi: 10.1093/petrology/egl013.

Jordan, T., Isacks, B., Allmendinger, R., Brewer, J., Ramos, V., and Ando, C. (1983). Andean tectonics related to geometry of subducted Nazca plate. GSA Bulletin, 94, 341-361.

Laeger, K., Halama, R., Hansteen, T., Savov, I.P., Murcia, H.F., Cortés, G.P., and Garbe-Schönberg, D. (2013). Crystallization conditions and petrogenesis of the lava dome from the $\sim 900$ years BP eruption of Cerro Machín Volcano, Colombia. Journal of South American Earth Sciences, 48, 193-208. doi: 10.1016/j.jsames.2013.09.009.

Leake, B., Woolley, A., Arps, C., Birch, W., Gilbert, M., Grice, J., Hawthorne, E., Kato, A., Kisch, H.J., Krivovichev, V.G., Linthout, K., Laird, J., Mandarino, J., Maresch, W.V., Nickel, E.H., Rock, N.M.S., Schumacher, J.C., Smith, D.C., Stephenson, N.C.N., Ungaretti, L., Whittaker, E.J.W., and Youzhi, G. (1997). Nomenclature of amphiboles: Report of the Subcommittee on Amphiboles of the International Mineralogical Association Commission on New Minerals and Mineral Names. European Journal of Mineralogy, 9(3), 623-651. doi: 10.1127/ ejm/9/3/0623.

Lescinsky, D. (1990). Geology, volcanology and petrology of Cerro Bravo, a young dacitic stratovolcano in west-central Colombia. MSc Thesis, Lousiana State University, Baton Rouge, USA.

Londoño, J. (2016). Evidence of recent deep magmatic activity at Cerro Bravo-Cerro Machín volcanic complex, central Colombia. Implications for future volcanic activity at Nevado del Ruiz, Cerro Machín and other volcanoes. Journal of Volcanology and Geothermal Research, 324, 156-168. doi: 10.1016/j. jvolgeores.2016.06.003.

Maksimov, A. (2009). The influence of water on the temperature of amphibole stability in melts. Journal of Volcanology and Seismology, 3(1), 27-33. doi: 10.1134/S0742046309010035.

Martel, C., Pichavant, M., Holtz, F., Scaillet, B., Bourdier, J., and Traineau, H. (1999). Effects of $f_{\mathrm{O} 2}$ and $\mathrm{H}_{2} \mathrm{O}$ on andesite phase relations between 2 and 4 kbar. Journal of Geophysical Research: Solid Earth, 104(B12), 29453-29470. doi: 10.1029/1999JB900191. 
Martínez, T., Valencia, R., Ceballos, H., Narváez, M., Pulgarín, A., Correa, T., Navarro, A., Murcia, A., Zuluaga, M., Rueda, G., y Pardo, V. (2014). Geología y estratigrafía del Complejo Volcánico Nevado del Ruiz. Informe final, Bogotá-Manizales - Popayán. Servicio Geológico Colombiano.

Mejía, E., Velandia, F., Zuluaga, C., López, J., y Cramer, T. (2012). Análisis estructural al noreste del volcán Nevado del Ruíz, Colombia - Aporte a la exploración geotérmica. Boletín de Geología, 34(1), 27-41.

Monsalve, M.L. (1991). Mapa preliminar de amenaza volcánica del volcán Cerro Bravo. INGEOMINAS. Manizales.

Monsalve, M.L., y Nuñez, A. (1992). El volcán Cerro Bravo, geología y amenaza volcánica. Revista INGEOMINAS, 1, 2-9.

Morimoto, N. (1989). Nomenclature of pyroxenes. Mineralogical Journal, 14(5), 198-221. doi: 10.2465/minerj.14.198.

Murcia, H., Borrero, C., and Németh, K. (in press). Overview and plumbing system implications of the monogenetic volcanism in the northernmost Andes' volcanic province. Journal of Volcanology and Geothermal Research. doi: 10.1016/j. jvolgeores.2018.06.013.

O’Neill, H., and Pownceby, M. (1993). Thermodynamic data from redox reactions at high temperatures. I. An experimental and theoretical assessment of the electrochemical method using stabilized zirconia electrolytes, with revised values for the Fe-“FeO”, Co-CoO, Ni-NiO and $\mathrm{Cu}-\mathrm{Cu}_{2} \mathrm{O}$ oxygen buffers, and new data for the $\mathrm{W}-\mathrm{WO}_{2}$ buffer. Contributions to Mineralogy and Petrology, 114(3), 296-314. doi: 10.1007/BF01046533.

Putirka, K.D. (2008). Thermometers and barometers for volcanic systems. Reviews in Mineralogy and Geochemistry, 69(1), 61-120.

Rahman, S., and MacKenzie, W. (1969). The crystallization of ternary feldspars: a study from natural rocks. American Journal of Science, 267, 391-406.

Rayo-Rocha, L. (2012). Evolución geoquímica y térmica del volcán Nevado del Ruiz, Colombia.
Tesis de Magister, Universidad Nacional de Colombia, Bogotá, Colombia.

Rayo-Rocha, L., y Zuluaga, C. (2011). Procesos magmáticos en el volcán Nevado del Ruiz: Un análisis cuantitativo textural. Boletín de Geología, 33(2), 59-72.

Ridolfi, F., Renzulli, A., and Puerini, M. (2010). Stability and chemical equilibrium of amphibole in calc-alkaline magmas: an overview, new thermobarometric formulations and application to subduction-related volcanoes. Contributions to Mineralogy and Petrology, 160(1), 45-66. doi: 10.1007/s00410-009-0465-7.

Rutherford, M., and Devine, J. (1988). The May 18, 1980, eruption of Mount St. Helens, 3, Stability and chemistry of amphibole in the magma chamber. Journal of Geophysical Research: Solid Earth, 93(B10), 11949-11959. doi: 10.1029/ JB093iB10p11949.

Rutherford, M., and Hill, P. (1993). Magma ascent rates from amphibole breakdown: an experimental study applied to the 1980-1986 Mount St. Helens eruptions. Journal of Geophysical Research: Solid Earth, 98(B11), 19667-19685. doi: 10.1029/93JB01613.

Rutherford, M., Sigurdsson, H., Carey, S., and Davis, A. (1985). The May 18, 1980, eruption of Mount St. Helens: 1. Melt composition and experimental phase equilibria. Journal of Geophysical Research: Solid Earth, 90(B4), 2929-2947. doi: 10.1029/JB090iB04p02929.

Sekine, T., Katsura, T., and Aramaki, S. (1979). Water saturated phase relations of some andesites with application to the estimation of initial temperature and water pressure at the time of eruption. Geochimica et Cosmochimica Acta, 43(8), 13671376.

Shcherbakov, V., Plechov, P., Izbekov, P., and Shipman, J. (2011). Plagioclase zoning as an indicator of magma processes at Bezymianny Volcano, Kamchatka. Contributions to Mineralogy and Petrology, 162(1), 83-99. doi: 10.1007/s00410010-0584-1.

Singer, B., Dungan, M., and Layne, G. (1995). Textures and $\mathrm{Sr}, \mathrm{Ba}, \mathrm{Mg}, \mathrm{Fe}, \mathrm{K}$, and $\mathrm{Ti}$ compositional 
profiles in volcanic plagioclase: clues to the dynamics of calc-alkaline magma chambers. American Mineralogist, 80(7-8), 776-798. doi: 10.2138/am-1995-7-819.

Stechern, A., Just, T., Holtz, F., Blume-Oeste, M., and Namur, O. (2017). Decoding magma plumbing and geochemical evolution beneath the Lastarria volcanic complex (Northern Chile). Evidence for multiple magma storage regions. Journal of Volcanology and Geothermal Research, 338, 2545. doi: 10.1016/j.jvolgeores.2017.03.018.

Stern, C., Futa, K., and Muehlenbachs, K. (1984). Isotope and trace element data for orogenic andesites from the Austral Andes. In: R.S. Harmon, B.A. Barreiro (eds.). Andean Magmatism (pp. 3146). Birkhäuser Boston.

Thouret, J. (1989). Geomorfología y crono-estratigrafía del Macizo Volcánico Ruiz-Tolima (Cordillera Central Colombiana). En: T. van der Hammen, S. Díaz-Piedrahita, V.J. Alvarez (eds.). Estudios de ecosistemas tropandinos, La Cordillera Central Colombiana, Transecto Parque Los Nevados (pp. 257-277). Berlin.

Thorpe, R., Francis, P., and O’Callaghan, L. (1984). Relative roles of source composition, fractional crystallization and crustal contamination in the petrogenesis of Andean volcanic rocks. Philosophical Transactions of the Royal Society a Mathematical, Physical and Engineering Sciences, 310(1514), 675-692. doi: 10.1098/rsta.1984.0014.

Tsuchiyama, A. (1985). Dissolution kinetics of plagioclase in the melt of the system diopsidealbite-anorthite, and origin of dusty plagioclase in andesites. Contributions to Mineralogy and Petrology, 89(1), 1-16. doi: 10.1007/BF01177585.

Vernon, R., Johnson, S., and Melis, E. (2004). Emplacement-related microstructures in the margin of a deformed pluton: the San José tonalite, Baja California, México. Journal of Structural Geology, 26(10), 1867-1884. doi: 10.1016/j. jsg.2004.02.007.

Vesga, C., y Barrero, D. (1978). Edades K/Ar en rocas ígneas y metamórficas de la Cordillera Central de Colombia y su implicación geológica. II Congreso Colombiano de Geología, Bogotá, Colombia.

Villagómez, D., and Spikings, R. (2013). Thermochronology and tectonics of the Central and Western Cordilleras of Colombia: Early Cretaceous-Tertiary evolution of the Northern Andes. Lithos, 160-161, 228-249. doi: 10.1016/j. lithos.2012.12.008.

Villagómez, D., Spikings, R., Magna, T., Kammer, A., Winkler, W., and Beltrán, A. (2011). Geochronology, geochemistry and tectonic evolution of the Western and Central cordilleras of Colombia. Lithos, 125(3-4), 875-896. doi: 10.1016/j.lithos.2011.05.003.

Whitney, D., and Evans, B. (2010). Abbreviations for names of rock-forming minerals. American Mineralogist, 95(1), 185-187. doi: 10.2138/ am.2010.3371.

\begin{tabular}{c} 
Camilo Pinzón \\
ORCID: 0000-0002-7897-4361 \\
Juan Felipe Echeverri \\
ORCID: 0000-0002-5510-6748 \\
Hugo Murcia \\
ORCID: 0000-0002-3570-1988 \\
Dayana Schonwalder Ángel \\
ORCID: 0000-0002-4653-1566 \\
\hline \hline
\end{tabular}

Trabajo recibido: marzo 02 de 2018

Trabajo aceptado: julio 24 de 2018 